\title{
COMPARISON OF SAND-II AND FERRET
}

\author{
NASTER
}

\section{Hanford Engineering Development Laboratory}

\author{
D.W. Wootan
}

F. Schmittroth

January 1981

This book was prepared as an account of work sponsored by an acency ot the United States Government Netrher the United States Government nor any agency thereof nor any of therr employees makes dny watranity express of mplied or dssumes any legall liability or responsiblity for the accuracy

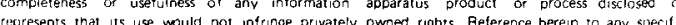

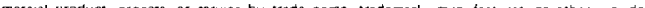

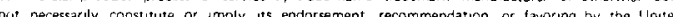

necessarily state or reflect those of the Unted States Government or any agency thereot 


\section{DISCLAIMER}

This report was prepared as an account of work sponsored by an agency of the United States Government. Neither the United States Government nor any agency Thereof, nor any of their employees, makes any warranty, express or implied, or assumes any legal liability or responsibility for the accuracy, completeness, or usefulness of any information, apparatus, product, or process disclosed, or represents that its use would not infringe privately owned rights. Reference herein to any specific commercial product, process, or service by trade name, trademark, manufacturer, or otherwise does not necessarily constitute or imply its endorsement, recommendation, or favoring by the United States Government or any agency thereof. The views and opinions of authors expressed herein do not necessarily state or reflect those of the United States Government or any agency thereof. 


\section{DISCLAIMER}

Portions of this document may be illegible in electronic image products. Images are produced from the best available original document. 
COMPARISON OF SAND-II AND FERRET

D. W. Wootan and F. Schmittroth

\begin{abstract}
A comparison was made of the advantages and disadvantages of two codes, SAND-II and FERRET, for determining the neutron flux spectrum and uncertainty from experimental dosimeter measurements as anticipated in the FFTF Reactor Characterization Program. This comparison involved an examination of the methodology and the operational performance of each code. The merits of each code were identified with respect to theoretical basis, directness of method, solution uniqueness, subjective influences, and sensitivity to various input parameters. The FERRET code represents a significant technical advance over SAND-II due to its stronger theoretical foundation, greater flexibility in handling multiple sets of data and incorporating other spectmom measurements such as proton recoil, improved treatment in the handling of uncertainty information through the use of coveriance matrices, and the fact that the credibility of the solution can be inferred directly from the credibility of the input data.
\end{abstract}


Abstract

List of Figures iii

List of Tables v v

I. Introduction 1

II . Code Descriptions 1

III. Comparison of Methods 6

IV. Comparison of Performance 8

A. Realistic Test Case 11

B. Sensitivity to Input Parameters 25

C. Simple Cases to Check Uncertainty Calculations 33

D. Summary of Performance Test Results 35

V. Conclusions 36

$\begin{array}{lll}\text { VI } & \text { References } & 37\end{array}$

Appendix A. Wel1- and I11-Determined Components of an 39 Unfolded Neutron Spectrum

Appendix B. Dosimeter Response Functions in EBR-II 42 Spectrum 


\section{LIST OF FIGURES}

Figure

SAND-II and FERRET solution spectra for EBR-II midplane location using a 11 reactions

2 Ratio of solution spectrum to input spectrum for SAND-II and FERRET using all reactions

3 SAND-II and FERRET solution spectrum uncertainty using all reactions

4 SAND-II and FERRET solution spectra using all reactions 16 except ${ }^{58} \mathrm{Fe}(n, \gamma)$

5 Ratio of solution spectrum and input spectrum for SAND-II and FERRET using a 11 reactions except ${ }^{58} \mathrm{Fe}(n, \gamma)$

6 SAND-II and FERRET solution spectrum uncertainty using al1 reactions except ${ }^{58} \mathrm{Fe}(n, \gamma)$

7 Ratio of solution snectrum to input spectrum for SAND-II and FERRET using a 11 reactions except ${ }^{45} \operatorname{Sc}(n, y)$

SAND-II and FERRET solution spectrum uncertainty using a11 reactions except ${ }^{45} \mathrm{Sc}(n, \gamma)$

9 Ratio of solution spectrum and input spectrum for SAID-II and FERRET using only ${ }^{46} \mathrm{Ti}(n, p)$

10 SAID-II and FERPET solution spectrum uncertainty using only ${ }^{46} \mathrm{Ti}(n, p)$

11 SAID-II solution snectrum variations with the number of iterations

12 Ratio of solution spectrum to input spectrum with variations in the number of iterations

13 SAND-II solution uncertainty variations with the number of Monte arlo recycles

14 Ratio of solution spectrum to input spectrum for FERRET with variations in the input spectrum short range correlations

15 FERRET solution spectrum uncertainty with variations in input spectrum short-range correlations of the reaction cross section correlations

A1 Decomposed components of unfolded neutron spectra 40

B1 Activation reaction response in EBR-II midplane

location. Product of cross section and flux spectrum, normalized to unity area 


\section{LIST OF FIGURES}

(continued)

\section{Figure}

B2

B3
Threshold reaction response in EBR-II midplane Fission reaction response in EBR-II midplane location
Page

44

45 


\section{LIST OF TABLES}

Table

1 Experimental Activities and Uncertainties

Page

2 SAND-II Cross Section Uncertainties in GAVE Format

9

3 Results of Simplified SAND-II Uncertainty Problems

10

35 


\section{COMPARISON OF SAND-II AND FERRET}

\section{INTRODUCTION}

A vital part of the Reactor Characterization Program of the Fast Flux Test Facility (FFTF) is the determination of the neutron flux spectra from experimental reaction rates in irradiated activation and fission detectors. At presend there are two HEDL computer codes, SAND-II and FERRET, that may be used for this task. FERRET is a newly developed, generalized least-squares data-adjustment code, that makes use of full covariance matrices to determine the "most likely" solution spectrum and uncertainty for a given set of input data. SAND-II is a widely used spectrum-unfolding code based on an iterative adjustment technique. It includes a Monte Carlo error analysis routine for generating uncertainty estimates on the final solution spectrum. Several developmental versions of the code exist at HEDL. In particular, work has been performed at HEDL to better understand the mathematical basis of the SAND-II algorithm, (1) and to improve the estimate of uncertainties associated with the solution spectrum. A technique has been devised to include a priori information in the analysis. ${ }^{1}$ ) Because this work is on-going and not widely documented, the present analysis is based on the fully documented version of the code. This study is a comparison of these two codes from both a technical and operational point of view. The technical aspect involves the method each code uses to arrive at a solution and estimate uncertainties. The operational aspect involves the performance of each code in selected test cases.

This study is one part of the program being carried out by Radiation and Shield Analysis under the Core Design and Nuclear Evaluation task.

\section{CODE DESCRIPTIONS}

\section{FERRET}

The FERRET code is a very general least-squares code oriented toward problems frequentiy encountered in nuclear data evaluations and reactor physics with strong emphasis on the proper utilization of important correlations and in providing quantitative uncertainty estimates. It was written to enable the 
user to apply it to a large variety of problems by modifying only the input subroutine. Some of the problems it has been applied to are damage function adjustments from integral measurements, simultaneous adjustment of reactor spectra and dosimeter cross sections based on both integral measurements and experimental proton-recoil spectra, and cross section evaluations based on simultaneous use of measured reaction rates, measured worths, microscopic measurements, and theoretical models. (2)

The method is based on a maximum-likelihood extension of generalized leastsquares methods that makes use of a log normal distribution. Only a brief sketch of the method is given here as a more complete documentation is provided el sewhere. $(2,3,4,5)$

Given a set of measured values $\left\{g_{m j}\right\} \equiv\left\{g_{m]}, g_{m 2}, \ldots\right\}$ along with the ir uncertainties and important correlations, assume that they are linearly related to a set of underlying parameters $\left\{w_{j}\right\} \equiv\left\{w_{1}, w_{2}, \ldots\right\}$. This linear relationship can be expressed by the Taylor series expansion

$$
\vec{g}(\vec{w}) \approx \vec{g}^{\star}+\vec{\nabla}_{w} \vec{g}^{\star} \cdot\left(\vec{w}-\vec{w}^{\star}\right)
$$

where

$$
\vec{g}^{\star}=\vec{g}\left(\vec{w}^{\star}\right)
$$

and $\vec{w}^{*}$ is the series expansion value. Letting

$$
\vec{f}_{m}=\vec{g}_{m}-\vec{g}^{\star}
$$

and

$$
\vec{x}=\vec{w}-\vec{w}^{\star}
$$

Then Eq. (1) has the usual form for the least-squares methods:

$$
\vec{f}_{m}=A \vec{x}
$$

where $A=\vec{\nabla}_{W} \vec{g}$ is the model matrix.

The problem is to combine the measured values $\left\{g_{m i}\right\}$ with the a priori values $\left\{w_{0 i}\right\}$ to obtain an adjusted or evaluated set of values for $\left\{w_{i}\right\}$. 
The FERRET algorithm is based on the minimization of

$$
s^{2}=s_{0}^{2}+s_{m}^{2}
$$

where in matrix notation

$$
s_{m}^{2}=\left(\vec{f}_{m}-A \vec{x}\right)^{\top} M_{g}^{-1}\left(\vec{f}_{m}-A \vec{x}\right)
$$

The a priori term is evaluated in a log space to ensure that the $\left\{w_{i}\right\}$ are positive

$$
s_{0}^{2}=\left(\vec{z}_{0}-\vec{z}\right)^{\top} M_{z 0}^{-1}\left(\vec{z}_{0}-\vec{z}\right)
$$

where

$$
z_{i}=\ln \left(w_{j}\right)
$$

The covariance matrix for the measurements is

$$
\left(M_{g}\right)_{i j}=\operatorname{Cov}\left(g_{m i}, g_{m j}\right)
$$

The covariance matrix for the a priori values is given by

$$
M_{z o}=\frac{\operatorname{Cov}\left(w_{0 i}, w_{0 j}\right)}{\left\langle w_{0 i}\right\rangle\left\langle w_{0 j}\right\rangle} .
$$

The choice of logarithmic variables necessitates iterating on $\vec{w}^{*}$ to obtain the minimum of Eq. (6).

Although the basic algorithm for the FERRET code can utilize any valid covariance matrix, a simple parameterization that allows construction of the needed covariance matrices from intuitive concepts is provided. The a priori fractional covariance matrix $\left(M_{z 0}\right)_{i j}$ is a sum of terms, $M_{i j}$, that represent various sources of uncertainty. The component matrices $M_{i j}$ are parameterized by

$$
M_{i j}=c^{2}+r_{i} r_{j} \rho_{i j}
$$


where

$$
\rho_{i j}=(1-\theta) \delta_{i j}+\theta e^{-\frac{(i-j)^{2}}{2 \gamma^{2}}}
$$

and

$$
\begin{aligned}
C= & \text { fractional normalization uncertainty } \\
r_{i}= & \text { fractional group-to-group uncertainty } \\
\theta= & \text { split between statistically independent uncertainties } \\
& \text { and short-range correlations } \\
\gamma= & \text { range of short-range correlation } .
\end{aligned}
$$

This allows a representation of $M_{i j}$ by three terms, a normalization uncertainty $C$, a set of statistically independent uncertainties $\left\{r_{i} \sqrt{1-\theta}\right\}$, and a shortrange correlation term with range $\gamma$.

$\underline{\text { SAND-II }}$

The SAND-II code was developed specifically for determining neutron flux spectra from multiple foil activation measurements. It is an established code with a tested library of cross sections. Different versions of the SAND-II code have been widely used and a broad base of experience has been built up over the years. Its primary application has been in providing consistent integral quantities such as total flux. The SAND-II, 620-group cross section 1 ibrary has been adjusted in shape and magnitude to give integrally consistent results in several well characterized reference spectra. (6) The SAND-II unfolding method is based on making iterative adjustments to a trial spectrum to obtain an appropriate fit between calculated and measured reaction rates. A Monte Carlo error analysis routine is included that provides uncertainty estimates for the derived flux spectrum. $A$ more complete documentation can be found elsewhere. $(7,8,9)$ The version of SAND-II described here was based on Reference 9 .

$$
\text { Let } \quad \begin{aligned}
& i=\text { detector index } \\
& j=\text { energy index } \\
& k=\text { iteration index } \\
& E_{j}=\text { lower energy bound of the } j^{\text {th }} \text { energy interval } \\
& m=\text { total number of energy intervals } \\
& n=\text { number of detectors used. }
\end{aligned}
$$


Assume an estimate of the flux spectrum is given $\left\{\phi_{j}^{[0]} ; j=1,2, \ldots, m\right\}$. Then for each iteration $k$ the calculated activity for the $i$ th detector reaction based on the $k^{\text {th }}$ iterative flux spectrum is

$$
A_{i}^{[k]}=\sum_{j=1}^{m} \sigma_{i j} \phi_{j}^{[k]}\left(E_{j+1}-E_{j}\right) .
$$

The total integral flux calculated from the $k^{\text {th }}$ iterative flux spectrum is

$$
\phi^{[k]}=\sum_{j=1}^{m} \phi_{j}^{[k]}\left(E_{j+1}-E_{j}\right) .
$$

Using

$$
\bar{\sigma}_{i}^{[k]}=\frac{A_{i}^{[k]}}{\phi^{[k]}}
$$

and

$$
\Phi=\frac{A_{i}}{[k]}=\frac{i}{\sigma_{i}^{[k]}}
$$

the total integral flux based on all detectors using the $k^{\text {th }}$ iterative flux spectrum is calculated by

$$
\Phi_{\Phi}^{[k]}=\frac{\sum_{i=1}^{n} w_{i}^{[k]_{\Phi}^{[k]}}}{\sum_{i=1}^{n} w_{i}^{[k]}} .
$$

The $w_{i}^{[k]}$ are weighting factors that include uncertainty information in the iterative procedure. The ratio of the measured to calculated activities is

$$
\mathrm{R}_{i}^{[\mathrm{k}]}=\frac{\phi^{[k]}}{\bar{\Phi}^{[k]}} \cdot \frac{\mathrm{A}_{i}}{A_{i}^{[k]}}
$$

and the $k^{\text {th }}$ iterative correction factor for the $j^{\text {th }}$ energy interval is

$$
c_{j}^{[k]}=\frac{\sum_{i=1}^{n} w_{i j}^{[k]} \ln R_{i}^{[k]}}{\sum_{i=1}^{n} w_{i j}^{[k]}}
$$


where $W_{i j}$ is a smoothing weight function based on moving linear averages that also incorporates uncertainty information.

Final1y,

$$
\phi_{j}^{[k+1]}=\phi_{j}^{[k]} \exp \left(c_{j}^{[k]}\right) .
$$

The Monte Carlo error analys is routine combines uncertainties in reaction rates and in reaction cross sections to determine the solution spectrum uncertainties. It selects values of reaction rates and cross sections with in assigned uncertainties for a preselected number of regular SAND-II runs, generates sets of solution spectra, and derives error estimates from these sets of spectra.

The cross section uncertainties are specified in a group averaged (GAVE) format. The entire energy range is separated into 15 broad group intervals. (9) Total correlation is assumed for the groups within a GAVE interval and there is no correlation between groups in different GAVE intervals. The uncertainty in each cross section is described by specifying an uncertainty in each GAVE interval.

\section{COMPARISON OF METHODS}

The dosimetry unfolding problem can be formulated as:

Given: 1. Some dosimetry cross sections with their uncertainties

2. An estimated neutron spectrum

3. Activation measurements performed in the actual neutron spectrum with the ir uncertainties.

What is the neutron spectrum and its uncertainty?

Since the number of energy groups is larger than the number of detectors, this problem is under-determined and has no unique solution. Stiffness or smoothness 
constraints must be applied for this type of problem to obtain useful solutions and uncertainties. One of the major differences between SAND-II and FERRET is in the method of specifying these constraints. These constraints are the major factor in determining the spectral shape and magnitude in regions of 1 ittle detector response, as illustrated in Appendix A.

The constraints in FERRET are specified by the user and consist of correlations and uncertainty information on the input spectrum in the form of a covariance matrix. Based on the covariance matrices for the input spectrum and the cross sections, and on the other available input information, FERRET provides the "most likely" solution spectrum and uncertainty. The short range correlations on the input spectrum provide a way to specify a certain a priori rigidity to the adjusted spectrum, so that neighboring points will be adjusted together. This prevents the formation of "unphysical" spectral structure that is not present in the a priori spectrum. Since the short range correlations and uncertainties reflect the degree of confidence placed in the a priori spectrum, some subjectivity is involved. However, there are techniques for explicitly calculating the covariance matrix associated with a reactor physics calculated spectrum, and it is possible to remove even this source of subjectivity. (10)

The assumptions on which the FERRET solution are based are explicitly specified by the user. Thus, the credibility of the solution can be inferred directly from the credibility of the input data. Subjectivity in the FERRET algorithm does not appear since it has a rigorous mathematical foundation that leads to a unique, most likely, solution and uncertainty.

On the other hand, no formal mathematical justification has been developed for the SAND-II iterative technique and it has not been proven that this technique must result in successively better iterative solutions. (7) The constraints in SAND-II include a smoothing weight function inherent in the algorithm and a limit on the number of iterations allowed. The iteration process in SAMD-II is terminated either by reaching the maximum number of iterations or by reaching the convergence criteria on the measured and calculated reaction rates, Subsidiary information or constraints required by SAND-II to obtain a unique solution and uncertainty include an iteration limit or convergence criteria, the number of Monte Carlo recycles to produce uncertainty estimates, and the number of points to be used in the smoothing weight function. The final SAND-II 
solution uncertainties do not reflect any uncertainties arising from these subsidiary conditions since no uncertainties are assigned to them. The SAND-II algorithm used in this study also does not take into account any a priori uncertainty information on the input flux spectrum. In some cases the effects of this subsidiary information can be observed by bracketing the best input values with values within an assumed uncertainty range. This is in sharp contrast to FERRET, where the needed subsidiary information (input covariances) is precisely stated by the user and is correctly propagated to the final result.

The new version of SAND-II (1) weights the input spectrum to provide a bound on the iterative spectra adjustments but so far does not provide valid uncertainty estimates for the solution spectrum.

\section{COMPARISON OF PERFORMANCE}

Comparison of SAND-II and FERRET from an operational point of view requires an analys is of their performance in selected problems. The problems selected for this analys is included simple problems that could be checked by hand and a realistic unfolding problem. Variations of the latter problem were used to illustrate specific points and to determine the sensitivity to the input parameters.

The realistic test case consisted of measured reaction rates with uncertainties and a reactor physics calculated flux spectrum for an EBR-II midplane elevation location at the core reflector interface. (11) This problem was chosen because it is a real life situation that is very similar to what will be done in the FTR core characterization program, and the FTR and EBR-II spectra possess many of the same spectral characteristics. The dosimeter reactions used, their measured saturated, infinitely dilute activities, and assigned uncertainties are given in Table 1.

The 47 group energy structure used in the reactor physics calculation of the input flux spectrum was chosen for the spectrum unfolding. Since the SAND-II version used normally uses 620 energy groups, slight changes had to be made in the program, primarily involving the input of the trial spectrum, to handle the 47 energy groups. 


\section{EXPERIMENTAL ACTIVITIES AND UNCERTAINTIES}

\begin{tabular}{|c|c|c|}
\hline Reaction & $\begin{array}{c}\text { Activity } \\
\text { (dis/sec/nucleus) }\end{array}$ & Uncertainty (\%) \\
\hline${ }^{59} \mathrm{Co}(n, \gamma)$ & $2.020 \times 10^{-10}$ & 3.0 \\
\hline${ }^{235} U(n, f)$ & $2.312 \times 10^{-9}$ & 3.2 \\
\hline${ }^{237} \mathrm{~Np}(n, f)$ & $6.270 \times 10^{-10}$ & 6.1 \\
\hline${ }^{45} \mathrm{Sc}(n, \gamma)$ & $3.752 \times 10^{-11}$ & 2.4 \\
\hline${ }^{54} \mathrm{Fe}(n, p)$ & $1.296 \times 10^{-11}$ & 1.7 \\
\hline${ }^{58} \mathrm{Fe}(n, \gamma)$ & $9.654 \times 10^{-12}$ & 1.3 \\
\hline${ }^{58} \mathrm{Ni}(n, p)$ & $1.738 \times 10^{-11}$ & 2.2 \\
\hline${ }^{46} \mathrm{Ti}(n, p)$ & $1.570 \times 10^{-12}$ & 1.2 \\
\hline
\end{tabular}

The only place where the group structure might have an effect on the SAND-II spectrum adjustments is in the use of the moving linear averages technique for determining the smoothing weight functions. This technique is based on an equal energy spacing assumption. ${ }^{(9)}$ The 620 groups are approximately logarithmically equispaced for $E<1 \mathrm{MeV}$ and approximately linearly equispaced for $E>1 \mathrm{MeV}$. The 47 groups are approximately logarithmically equispaced for $E<2 \mathrm{MeV}$ and are approximately linearly equispaced for E>2 MeV. The similarities between the 47 and 620 group structures and the fact that the SAND-II iteration procedure is relatively insensitive to the form of the smoothing weight function ${ }^{(9)}$ indicate that the use of 47 groups would have little effect on the performance of SAND-II. In addition, the available reactions do not possess enough spectral structure to resolve the spectrum as fine as the 620 groups require.

The 47 group reaction cross sections were collapsed from the ENDF/B-IV values using the calculated EBR-II spectrum. (11) The same cross section set, in different formats, was used for both FERRET and SAND-II. The cross section uncertainties and correlations were taken from a previous study $(12)$ in the FERRET format. The uncertainty values were converted to the GAVE format for use in SAND-II, and are shown in Table 2. 
SAND-II CROSS SECTION UNCERTAINTIES IN GAVE FORMAT

\begin{tabular}{|c|c|c|c|c|c|c|c|c|c|c|}
\hline \multirow{2}{*}{$\begin{array}{c}\text { GAVE } \\
\text { Interval } \\
\end{array}$} & \multicolumn{2}{|c|}{ Lower Bound } & \multicolumn{8}{|c|}{ Cross Section Uncertainty (percent) } \\
\hline & Group & Energy (MeV) & ${ }^{59} \mathrm{Co}(n, \gamma)$ & $235 U(n, f)$ & ${ }^{2{ }^{7}} \mathrm{~Np}(\mathrm{n}, \mathrm{f})$ & ${ }^{45} \operatorname{Sc}(n, \gamma)$ & ${ }^{54} \mathrm{Fe}(n, p)$ & ${ }^{58} \mathrm{Fe}(n, \gamma)$ & ${ }^{58} \mathrm{Ni}(\mathrm{n}, \mathrm{p})$ & ${ }^{46} \mathrm{Ti}(\mathrm{n}, \mathrm{p})$ \\
\hline 1 & 1 & $2.5300-008$ & 3.0 & 8.0 & 18.3 & 10.0 & 100.0 & 10.0 & 100.0 & 100.0 \\
\hline 2 & 2 & $1.1254-006$ & 10.4 & 9.2 & 20.4 & 10.0 & 100.0 & 14.7 & 100.0 & 100.0 \\
\hline 3 & 6 & $1.3710-005$ & 10.8 & 10.0 & 26.6 & 32.6 & 100.0 & 36.4 & 100.0 & 100.0 \\
\hline 4 & 22 & $1.5034-002$ & 21.8 & 7.7 & 24.3 & 35.0 & 100.0 & 40.1 & 100.0 & 100.0 \\
\hline 5 & 27 & $1.1109-001$ & 28.4 & 5.0 & 11.2 & 36.1 & 100.0 & 48.0 & 100.0 & 100.0 \\
\hline 6 & 32 & $6.3928-001$ & 28.4 & 5.0 & 9.9 & 36.1 & 100.0 & 54.2 & 78.9 & 100.0 \\
\hline 7 & 35 & $1.3834+000$ & 28.4 & 5.0 & 9.9 & 36.1 & 55.3 & 63.1 & 21.7 & 100.0 \\
\hline 8 & 37 & $2.2313+000$ & 30.7 & 5.0 & 9.9 & 37.8 & 9.4 & 69.2 & 9.4 & 61.7 \\
\hline 9 & 39 & $3.6788+000$ & 33.2 & 5.0 & 9.9 & 39.6 & 9.4 & 73.2 & 9.4 & 21.0 \\
\hline 10 & 40 & $4.9659+000$ & 36.1 & 5.4 & 9.9 & 41.7 & 9.4 & 77.5 & 9.4 & 51.3 \\
\hline 11 & 41 & $6.0653+000$ & 38.8 & 7.0 & 10.3 & 43.6 & 9.4 & 81.5 & 11.3 & 9.4 \\
\hline 12 & 42 & $7.4082+000$ & 41.1 & 8.2 & 11.2 & 45.2 & 17.9 & 84.8 & 14.2 & 13.9 \\
\hline 13 & 43 & $8.6071+000$ & 43.6 & 9.6 & 12.2 & 46.9 & 18.3 & 88.3 & 17.4 & 19.6 \\
\hline 14 & 45 & $1.1618+001$ & 46.9 & 11.3 & 13.5 & 49.1 & 18.9 & 93.3 & 18.8 & 17.4 \\
\hline 15 & 46 & $1.3499+001$ & 50.2 & 13.0 & 14.9 & 51.3 & 19.4 & 97.5 & 15.7 & 15.6 \\
\hline
\end{tabular}


The response of the reaction cross sections in the 47 group EBR-II spectrum, included in Appendix $B$, revealed that the ${ }^{59} \mathrm{Co}(n, \gamma)$ reaction had a large response in one group at $1 \times 10^{-4} \mathrm{MeV}$, the ${ }^{58} \mathrm{Fe}(\mathrm{n}, \gamma)$ reaction had a large response in two groups between $2 \times 10^{-4}$ and $4 \times 10^{-4} \mathrm{MeV}$, and the other responses were primarily between $6 \times 10^{-3}$ and $8 \mathrm{MeV}$. This reaction set provided regions of good and bad detector coverage so that the performance of the codes could be anaiyzed in both situations.

\section{A. REALISTIC TEST CASE}

The measured input activities and uncertainties, the reactor physics calculated input spectrum, and the other input parameters described in a later section were used to adjust the calculated EBR-II flux with both SAND-II and FERRET. Figure 1 shows the solution spectra from both codes along with the a priori input spectrum. It can be seen that the basic input spectral structure was retained in both solutions, that there are areas where the two solutions agree quite we 11 , and that there are areas where the two solutions differ significantly. The large dip around $2 \times 10^{-3} \mathrm{MeV}$ is due to the sodium resonance. The small dip around $2 \times 10^{-2} \mathrm{MeV}$ is due to the small energy width of this group in the 47 group structure and the fact that the integral group flux is being plotted. Figure 2 is a plot of the ratio of the solution flux to the input flux for both codes and shows the characteristics of the adjustment made by each code and the areas of agreement and disagreement in the solutions. The adjustments made by FERRET can be seen to be governed by the short range correlations on the input spectrum, while the adjustments made by SAND-II are characterized by several regions of sharp discontinuity. The solution spectra agree to $10 \%$ at $1 \times 10^{-4} \mathrm{MeV}$ and to better than $5 \%$ between $2 \times 10^{-2}$ and $6 \mathrm{MeV}$, two regions of large detector response. The solutions differ by about $40 \%$ between $4 \times 10^{-4}$ and $2 \times 10^{-2} \mathrm{MeV}$, by about $50 \%$ above $10 \mathrm{MeV}$, and by over $100 \%$ below $6 \times 10^{-6} \mathrm{MeV}$. The $10 \%$ deviation at the relatively isolated ${ }^{59} \mathrm{Co}(n, \gamma)$ response at $1 \times 10^{-4} \mathrm{MeV}$ was about the same as the uncertainty on the ${ }^{59} \mathrm{Co}(n, \gamma)$ cross section at this energy. The other differences in the spectra were generally less than the SAND-II uncertainty values.

Figure 3 shows the FERRET and SAND-II spectral uncertainties. It should be pointed out that the FERRET uncertainty values are the diagonal elements of the full covariance matrix and that, due to the correlations in the solution 


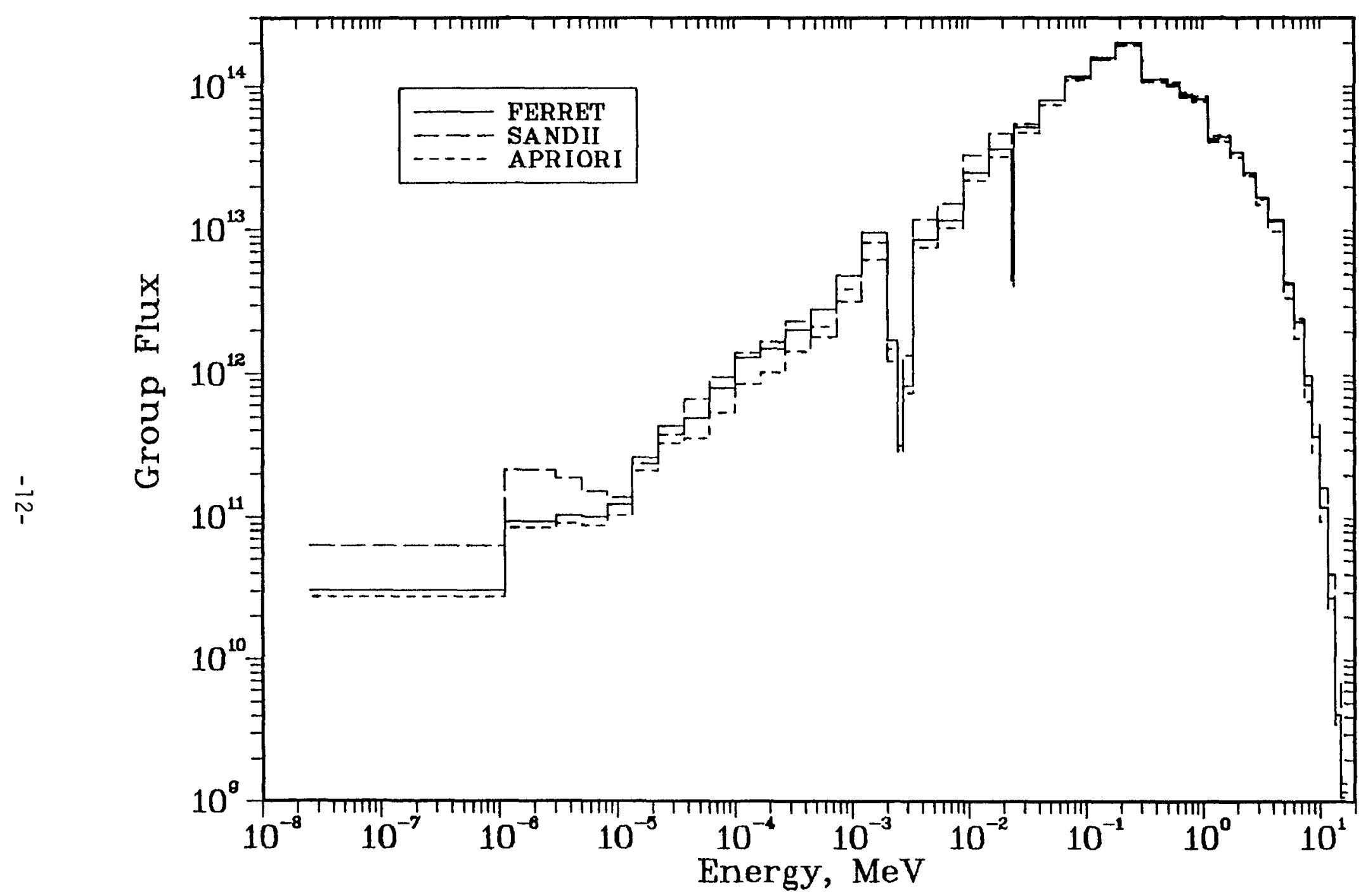

FIGURE 1. SAND-II and FERRET solution spectra for EBR-II midplane location using all reactions. 


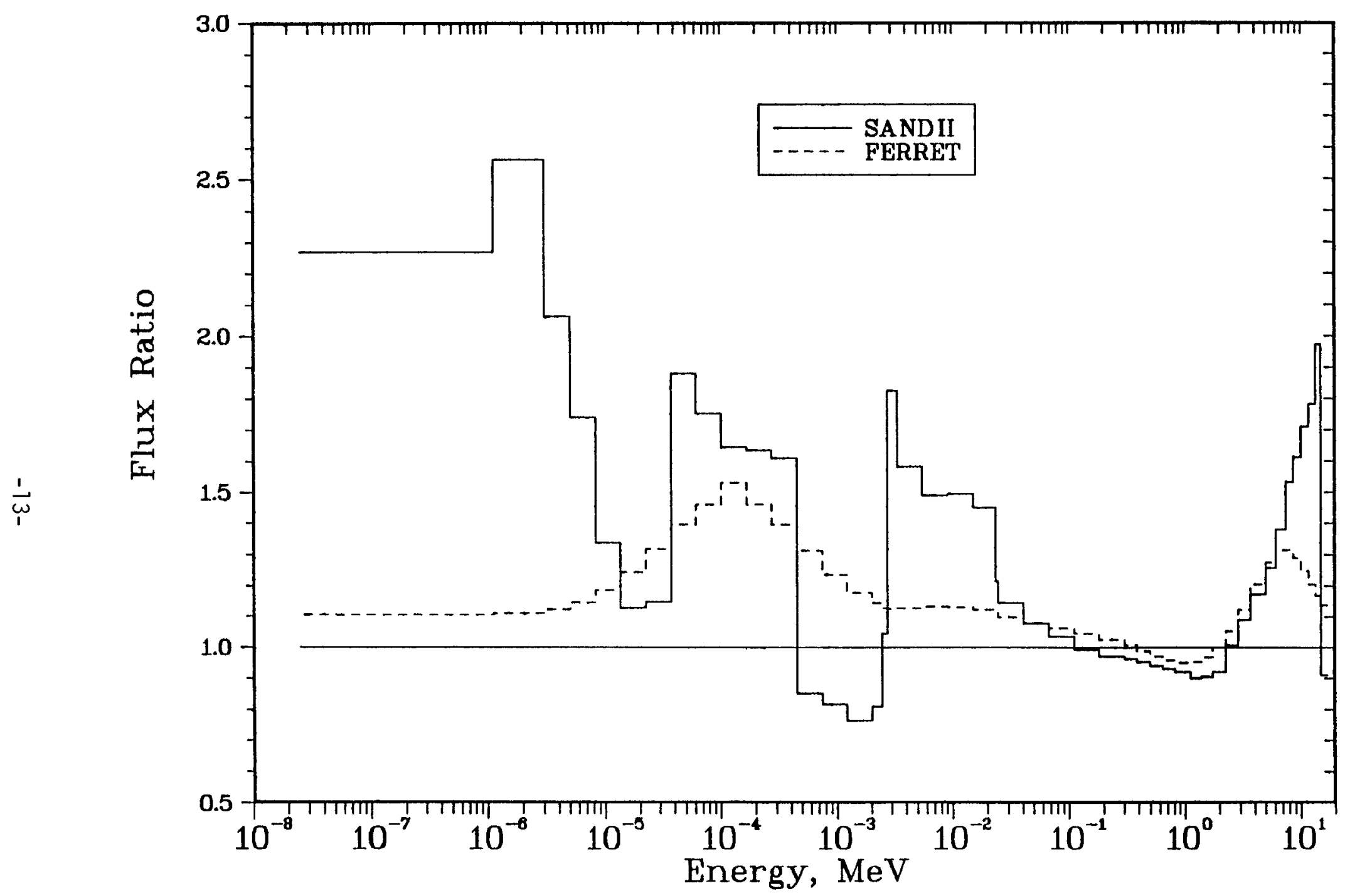

FIGURE 2. Ratio of solution spectrum to input spectrum for SAND-II and FERRET using all reactions. 


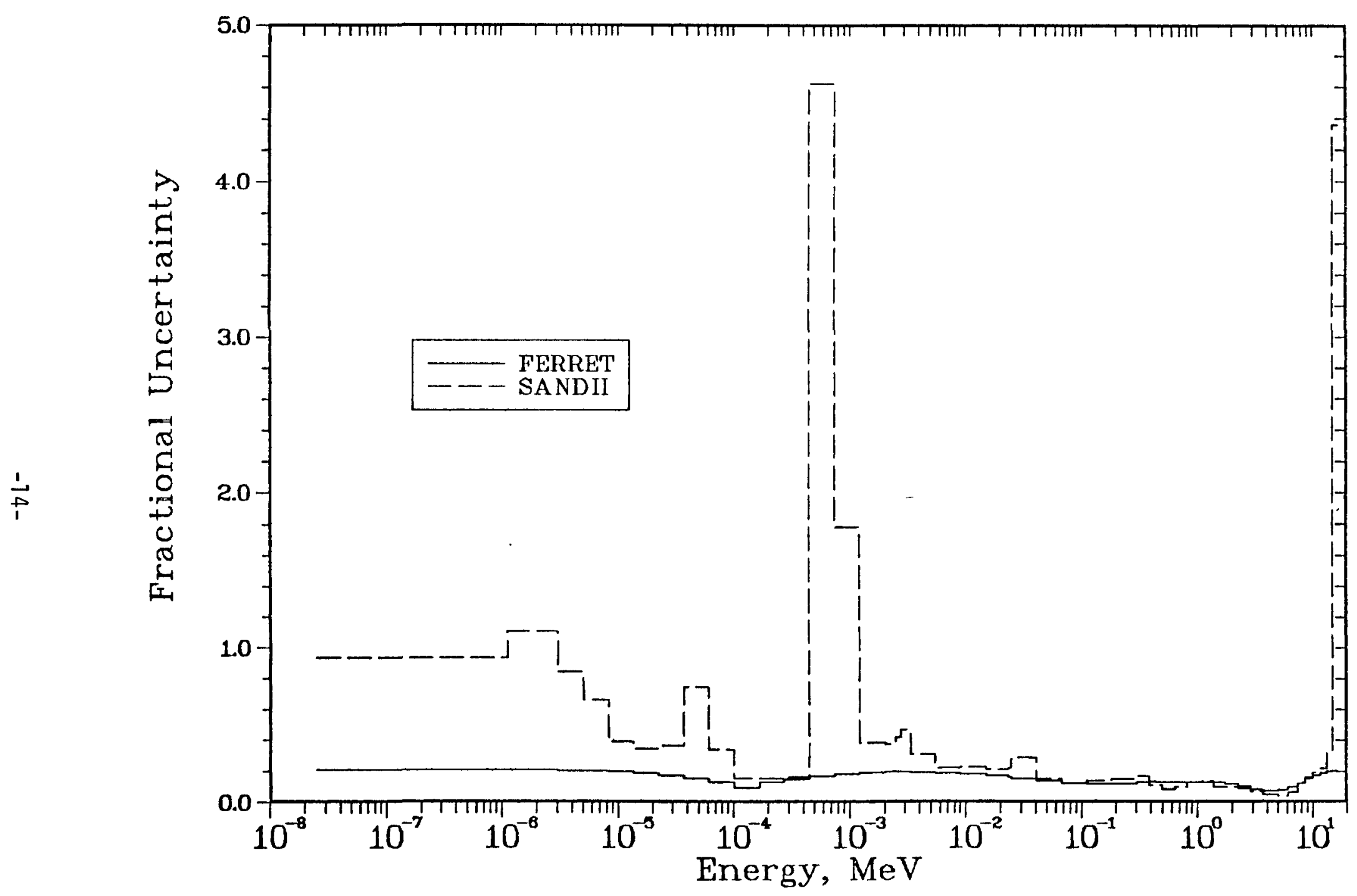

FIGURE 3. SAND-II and FERRET solution spectrum uncertainty using all reactions. 
spectrum, integral quantities calculated with the full covariance matrix may have uncertainties that are much lower. The FERRET uncertainties reflected the spectrum correlations, with the decrease in uncertainty in the regions of large response influencing the uncertainty in neighboring groups. The SAND-II uncertainties were much less correlated and were unrealistically high in several groups. The uncertainty in one group around $5 \times 10^{-4} \mathrm{MeV}$ was given as over $400 \%$ by SAND-II. Although the basic shapes of the uncertainty plots follow the same trend, the agreement in the uncertainty at the response peaks was less than anticipated. Examination of the Monte Carlo recycle information revealed that several of the recycle solutions were far from converged and that the deviation of measured to calculated activities for the ${ }^{58} \mathrm{Fe}(n, \gamma)$ reaction was as much as $140 \%$. A possible explanation for this behavior is that the Monte Carlo error analysis routine was making random adjustments in the ${ }^{58} \mathrm{Fe}$ cross section that resulted in divergent solutions and consequently large uncertainty values in the regions of little detector response.

This explanation was investigated by running the same problem with the ${ }^{58} \mathrm{Fe}(n, \gamma)$ reaction removed. The results are shown in Figures 4,5 and 6 . A comparison of Figures 4 and 5 with Figures 1 and 2 indicate that there was almost no change in the solution spectra. A comparison of Figure 6 with Figure 3 , however, reveals dramatic changes in the SAND-II uncertainty, while the FERRET uncertainty was nearly identical to that with the ${ }^{58} \mathrm{Fe}(n, \gamma)$ reaction included. The extremely large SAND-II uncertainty values were not present and there was very good agreement in uncertainty from both codes in the areas of $10^{-4}, 10^{-1}$, and $1 \mathrm{MeV}$. These areas correspond to the peaks of the response functions for the ${ }^{59} \mathrm{Co}(n, \gamma),{ }^{235} \mathrm{U}(n, f)$, and ${ }^{237} \mathrm{~Np}(n, f)$ reactions, respectively. The large dip in the uncertainty for both codes around $4 \mathrm{MeV}$ corresponds to the overlapping peak responses of the ${ }^{46} \mathrm{Ti}(n, p),{ }^{58} \mathrm{Ni}(n, p)$, and ${ }^{54} \mathrm{Fe}(n, p)$ threshold detectors. The factor of two difference in uncertainty between the two codes at this dip indicates a different treatment of the uncertainty in the case of these overlapping responses.

The SAND-II uncertainty values in the areas of little detector response still revealed some unphysical characteristics. There was a sharp dip in the SAND-II uncertainty around $2 \times 10^{-3} \mathrm{MeV}$ that did not correspond to any significant response from the detectors and was in fact at a peak in the FERRET uncertainty. Although the ${ }^{59} \mathrm{Co}(n, \gamma)$ response was 1 imited almost entirely to 


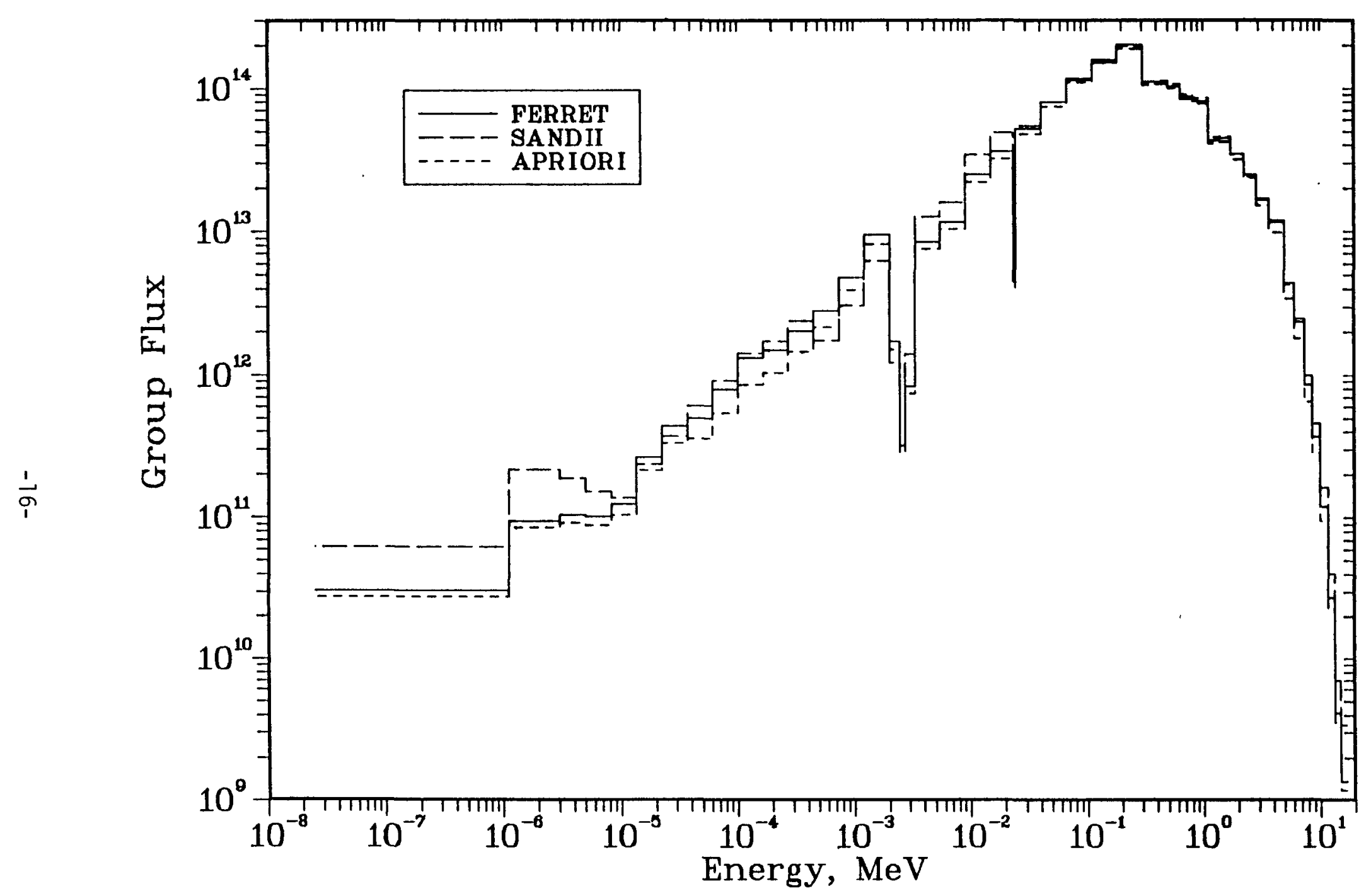

FIGURE 4. SAND-II and FERRET solution spectra using all reactions except ${ }^{58} \mathrm{Fe}(n, \gamma)$. 


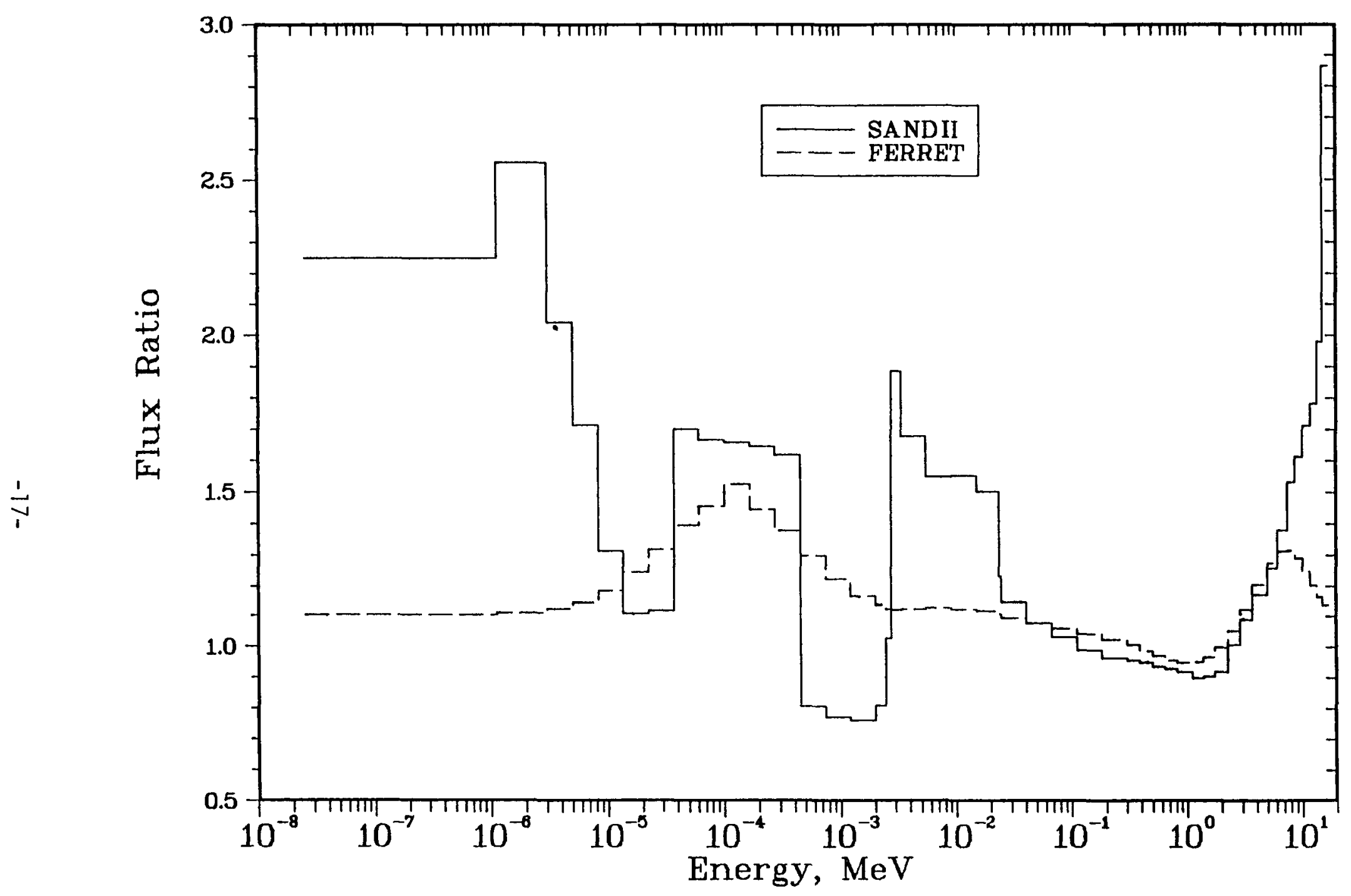

FIGURE 5. Ratio of solution spectrum and input spectrum for SAND-II and FERRET using all reactions except ${ }^{58} \mathrm{Fe}(n, \gamma)$. 


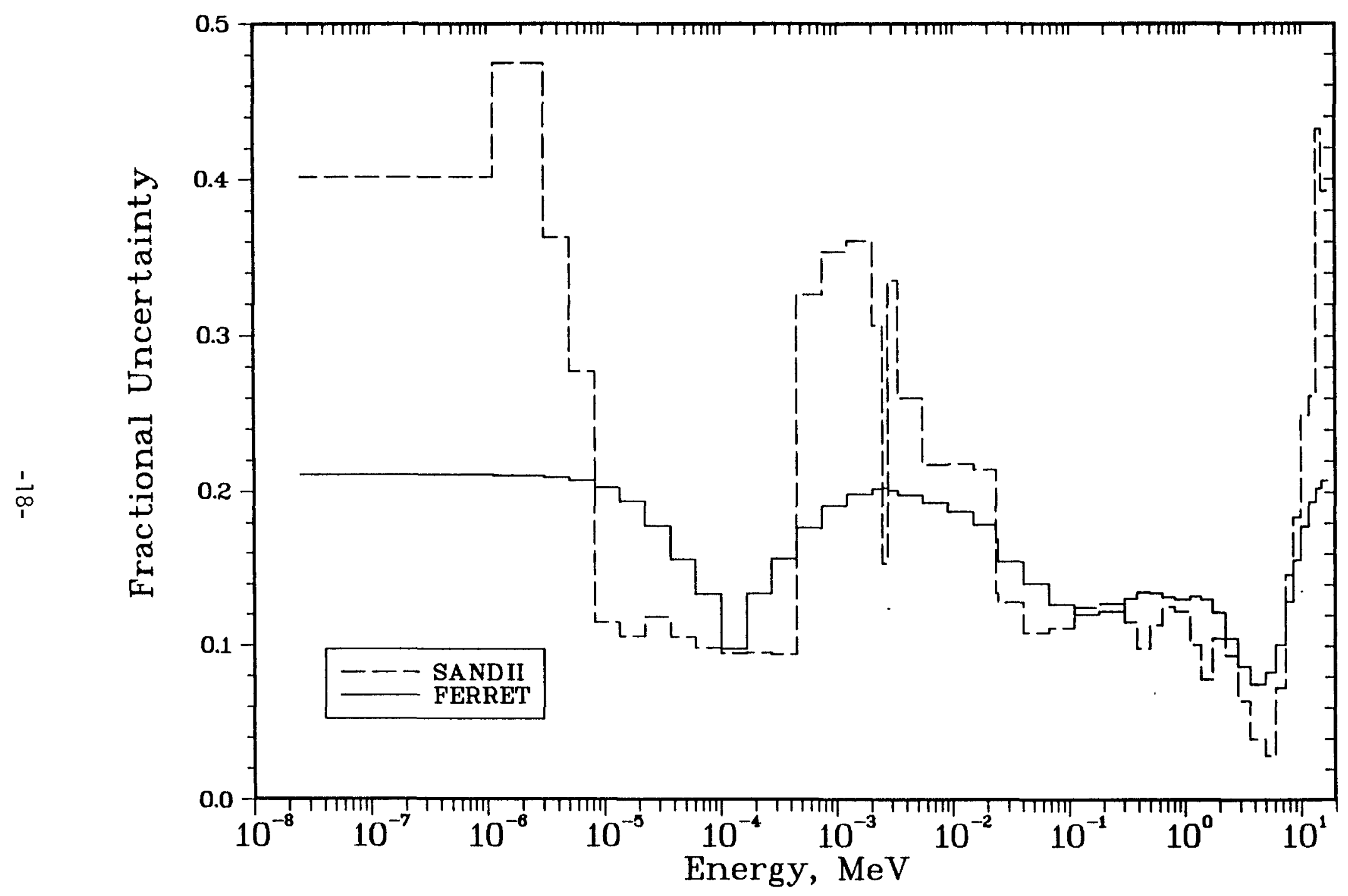

FIGURE 6. SAND-II and FERRET solution spectrum uncertainty using all reactions except ${ }^{58} \mathrm{Fe}(n, \gamma)$. 
one group at $10^{-4} \mathrm{MeV}$, SAND-II gave the same uncertainty from $1 \times 10^{-5}$ to $4 \times 10^{-4} \mathrm{MeV}$. Without the ${ }^{58} \mathrm{Fe}(n, \gamma)$ reaction, the only significant response in this energy range was from the narrow ${ }^{59} \mathrm{Co}(n, \gamma)$ response. Therefore, the low uncertainties given by SAND-II in this region are unjustified and unrealistic.

Since the removal of the ${ }^{58} \mathrm{Fe}(n, \gamma)$ reaction had such a dramatic effect on the SAND-II solution uncertainties, a different reaction was removed and the results observed. The ${ }^{58} \mathrm{Fe}(n, \gamma)$ reaction was put back in and a 71 of the reactions except the ${ }^{45} \mathrm{Sc}(n, \gamma)$ reaction were used to adjust the spectrum. The flux ratios and uncertainties are shown in Figures 7 and 8 . A comparison of the flux ratios in Figure 7 with those in Figure 2 revealed only slight changes in the FERRET solution, while the SAND-II solution was changed to be in closer agreement with FERRET below $10^{-5} \mathrm{MeV}$ and between $4 \times 10^{-4}$ and $2 \times 10^{-2} \mathrm{MeV}$. Since these changes did not occur when the ${ }^{58} \mathrm{Fe}(n, \gamma)$ reaction was removed, they can be attributed to the effect of the absence of the ${ }^{45} \mathrm{Sc}(n, \gamma)$ reaction on the SAND-II flux corrections. The removal of the ${ }^{45} \mathrm{Sc}(n, \gamma)$ reaction allowed a slightly better match of the other measured and calculated activities in SAND-II. The changes in the SAND-II solution spectrum were located in a region of overlapping response from the ${ }^{45} \mathrm{Sc}(n, \gamma),{ }^{58} \mathrm{Fe}(n, \gamma)$ and ${ }^{59} \mathrm{Co}(n, \gamma)$ reactions and indicated that there might be a slight inconsistency in the measured activities or cross sections of ${ }^{45} \mathrm{Sc}(n, \gamma)$ and ${ }^{59} \mathrm{Co}(n, \gamma)$. This is not a problem for FERRET since it can make minor adjustments in the cross sections within the assigned uncertainties and correlations in regions of overlapping response.

The solution uncertainties in Figure 8 show several interesting features. Although the FERRET uncertainty again remained relatively unchanged, the SAND-II uncertainty was reduced in all groups with the removal of the ${ }^{45} \mathrm{Sc}(n, \gamma)$ reaction. Dramatic reductions in uncertainty occurred below $3 \times 10^{-6} \mathrm{MeV}$ and at $3 \times 10^{-3} \mathrm{MeV}$. The uncertainty below $3 \times 10^{-6} \mathrm{MeV}$ dropped from around $100 \%$ to less than $12 \%$. Since the ${ }^{45} \mathrm{Sc}(n, \gamma)$ reaction was the only one with a noticeable response at that low an energy, it indicates that SAND-II may give unjustifiably low uncertainty estimates in regions of little or no response. This can also be seen in another region of little response at $3 \times 10^{-3} \mathrm{MeV}$, where the SAND-II uncertainty was reduced from $47 \%$ to $16 \%$ with the removal of the 


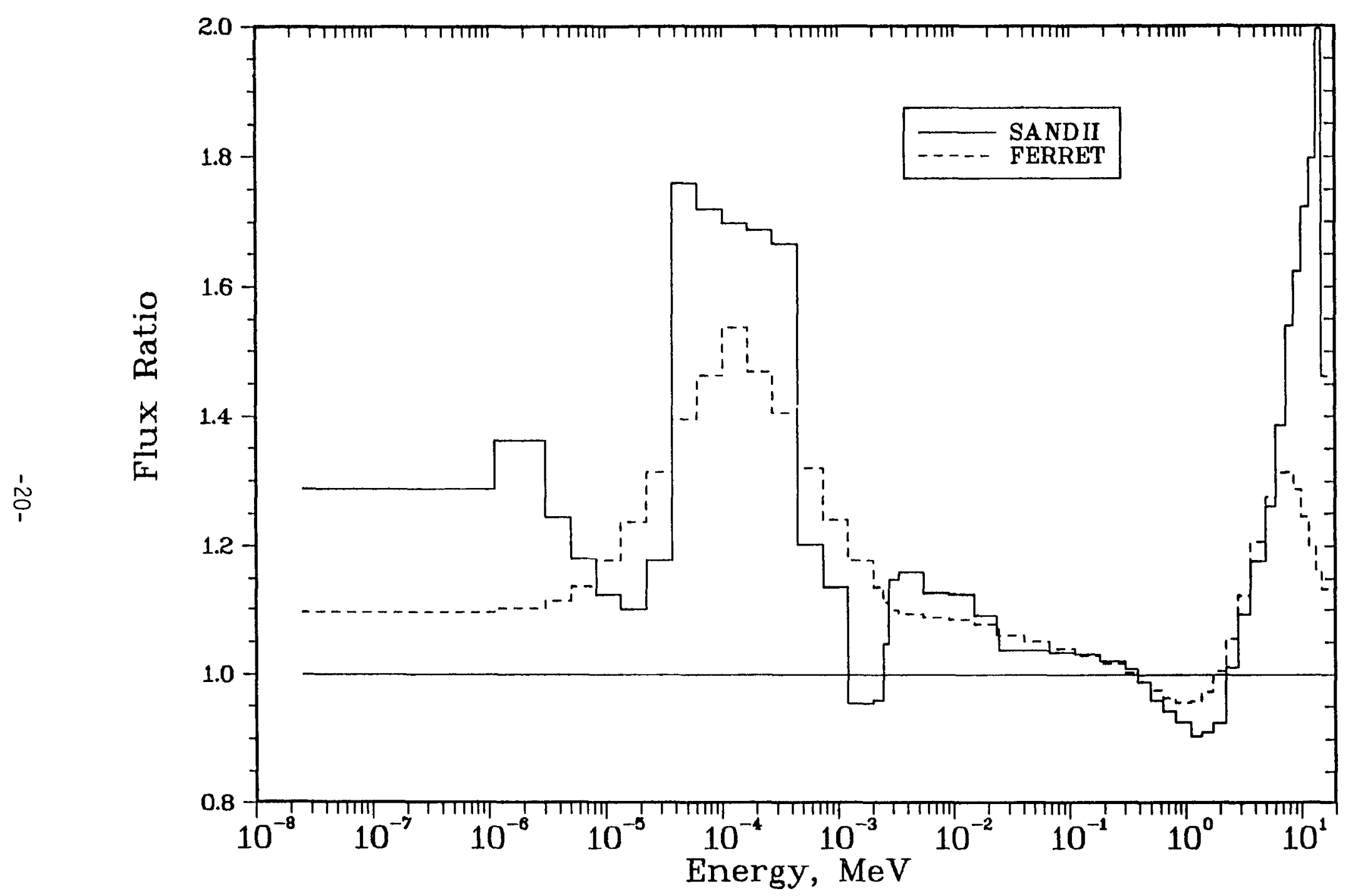

FIGURE 7. Ratio of solution spectrum to input spectrum for SAND-II and FERRET using all reactions except ${ }^{45} \mathrm{Sc}(n, \gamma)$. 


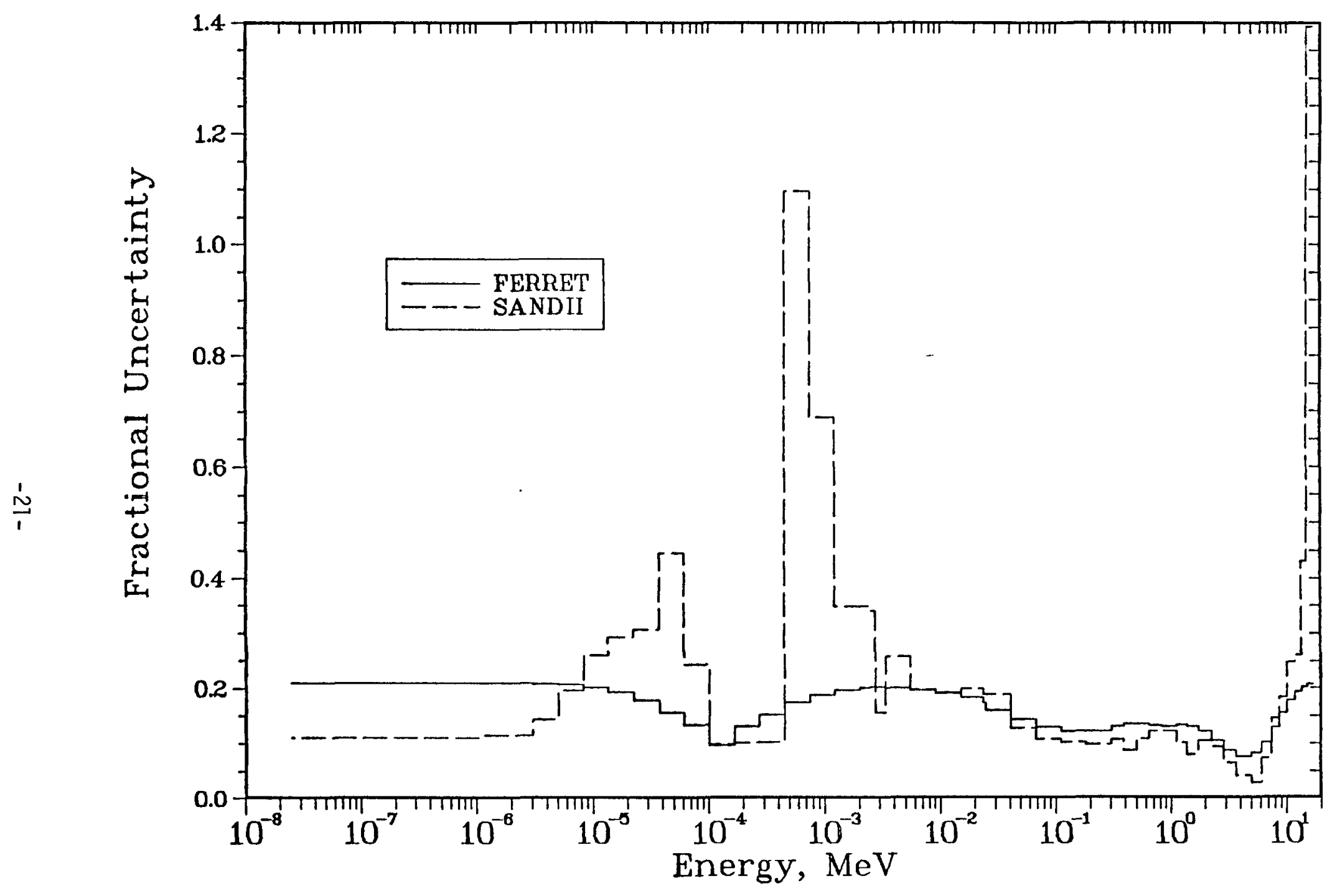

FIGURE 8. SAND-II and FERRET solution spectrum uncertainty using a 71 reactions except ${ }^{45} \mathrm{Sc}(n, \gamma)$. 
${ }^{45} \mathrm{Sc}(n, \gamma)$ reaction. The SAND-II uncertainty around $4 \times 10^{-4}$ MeV was again large, over $100 \%$, as compared to $30 \%$ when the ${ }^{58} \mathrm{Fe}(n, \gamma)$ reaction was removed, but was still much less than the $400 \%$ uncertainty with all of the reactions.

Some of the effects indicated in the previous examples were confirmed by using only a single reaction. Although this kind of case is not run in practice, the example does isolate a difference between SAND-II and FERRET. The ${ }^{46} T i(n, p)$ threshold reaction was used alone to adjust the solution spectrum and uncertainty. The rest of the input information was the same, except that a constant $10 \%$ cross section uncertainty was used. The response of the ${ }^{46} \mathrm{Ti}(n, p)$ reaction is limited to a fairly small energy range, with no response outside of this range. The solution flux ratios and uncertainties are shown in Figures 9 and 10 . In this case, SAND-II applied a nearly constant correction factor to the entire spectrum. The flux magnitude outside the area of reaction response had no effect on the calculated activity since the cross section was essentially zero there. FERRET made a much smaller spectrum normalization in the region of no response based on the input normalization uncertainty on the spectrum. The FERRET spectrum was adjusted primarily in the region of reaction response to match up the measured and calculated activities. The extent of adjustment outside the response region depends on the choice of a priori spectrum correlation. Incorporation of a priori spectrum information in the newer version of SAND-II should reduce the spectrum adjustments in low response regions.

The uncertainty results in Figure 10 confirm that SAND-II may underpredict the uncertainty on the solution spectrum in regions of little or no response. SAND-II assigned a sma11, constant uncertainty to the entire spectrum, even though the reaction response was 1 imited to a small portion of the spectrum. The FERRET uncertainty was almost the same as the input uncertainty estimate except in the vicinity of the reaction response, where the input correlations and uncertainties on the spectrum, cross section, and activity reduce the spectrum uncertainty. While the FERRET uncertainty in the region of no response was based on the input flux uncertainty, the SAND-II uncertainty in this region was based on the uncertainty in the region of response. In practice, the SAND-I I uncertainty estimates in regions of little or no response may be approximated by bracketing the best input spectrum estimate with spectra within the uncertainty range. 


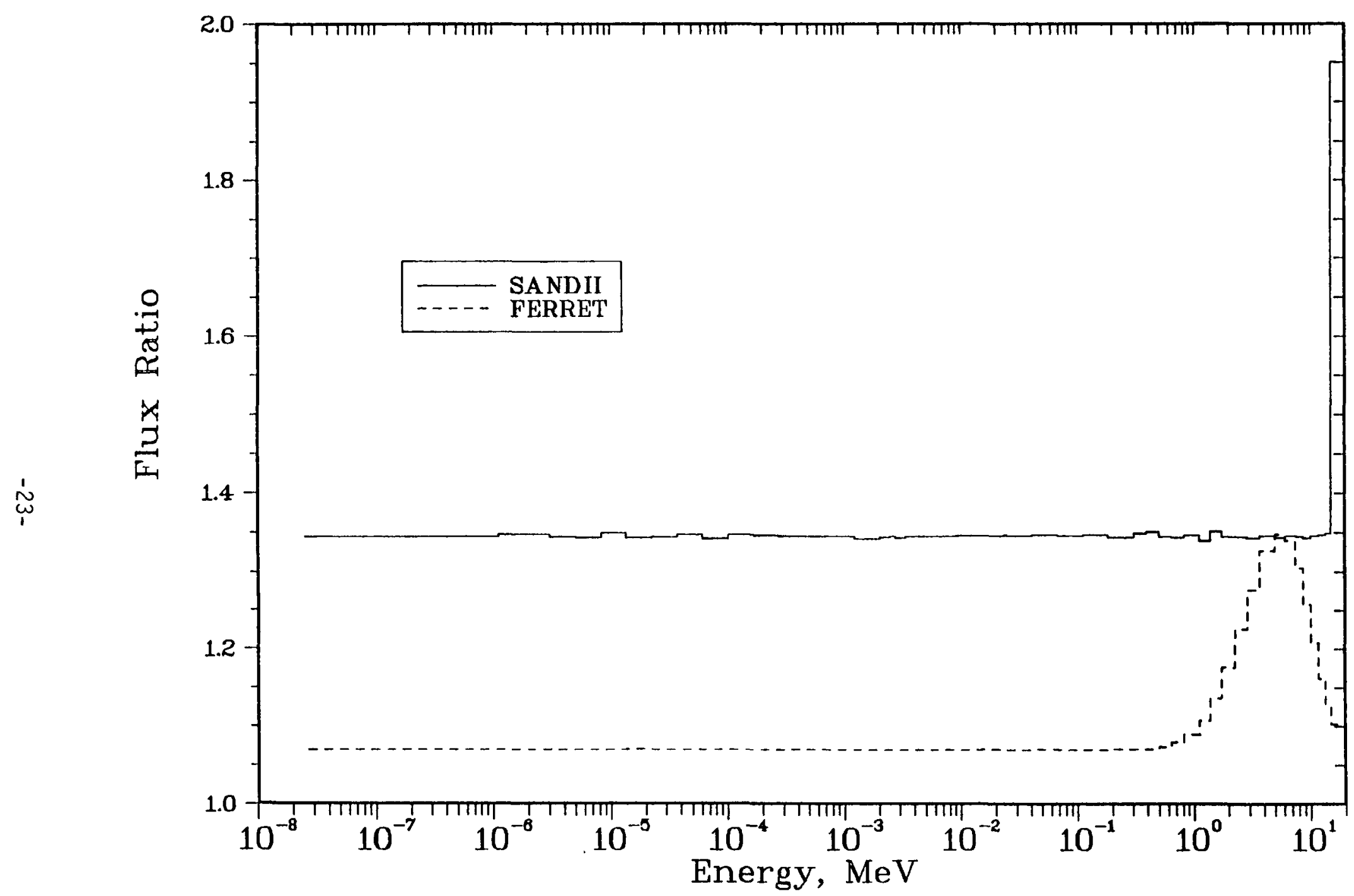

FIGURE 9. Ratio of solution spectrum and input spectrum for SAND-II and FERRET using only ${ }^{46} \mathrm{Ti}(n, p)$. 


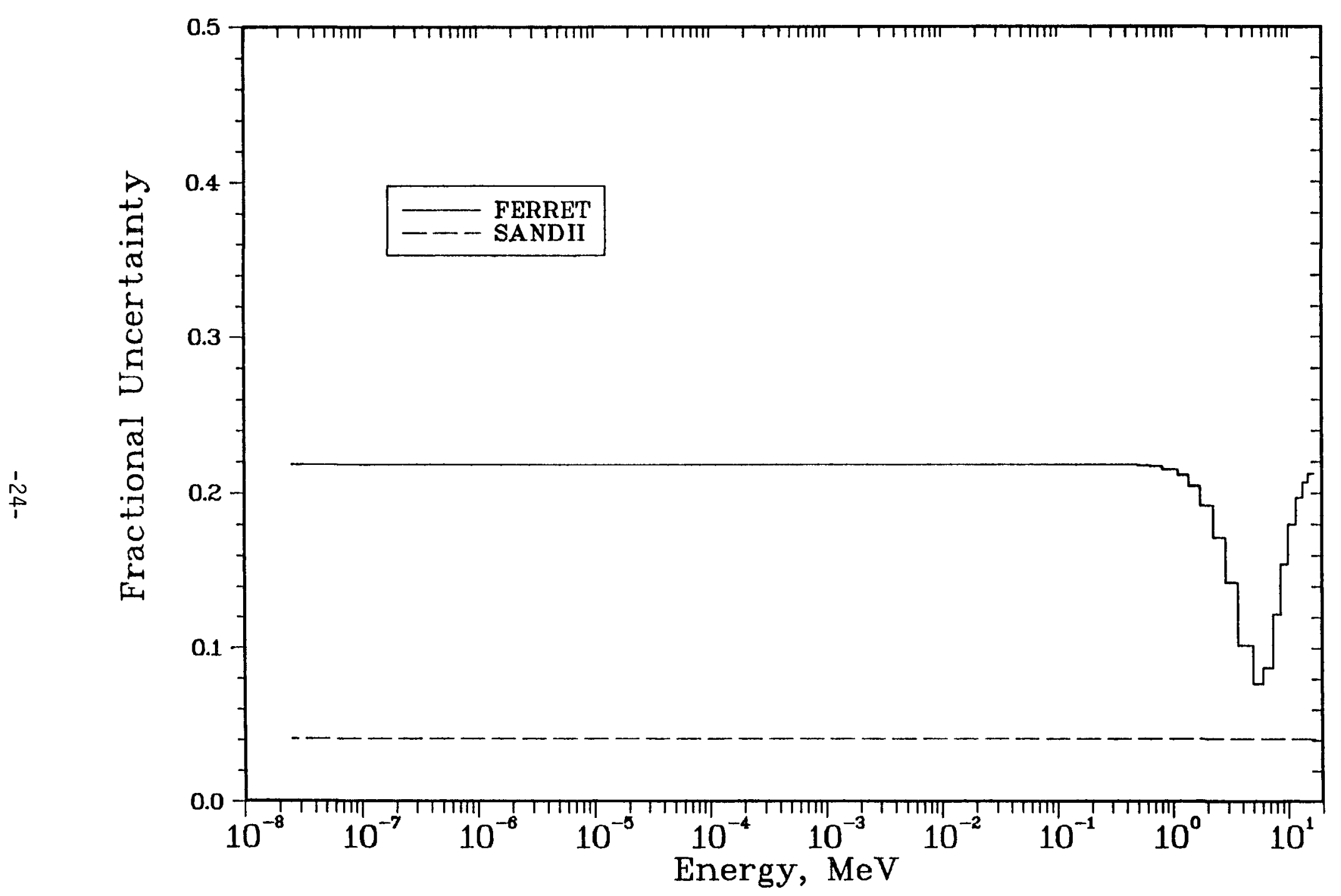

FIGURE 10. SAND-II and FERRET solution spectrum uncertainty using only ${ }^{46} \mathrm{Ti}(n, p)$. 


\section{B. SENSITIVITY TO INPUT PARAMETERS}

The amount of subjectivity present in the adjustment and uncertainty calculations can be inferred from the effects of variations of the major input parameters involved.

\section{SAND-II}

Subjective influences can be introduced into the SAND-II algorithm through the choice of input parameters such as the number of iterations and the number of Monte Carlo recycles. Figures 11 and 12 show the SAND-II solution spectra and flux ratios after 10,20 , and 40 iterations. The flux in regions of significant detector response remained relatively fixed, while the flux was varied in regions of little response in an attempt to better match the measured and calculated activities. Two regions of little response are below $10^{-5} \mathrm{MeV}$ and between $4 \times 10^{-4}$ and $3 \times 10^{-3} \mathrm{MeV}$. In the region below $10^{-5} \mathrm{MeV}$ and between $3 \times 10^{-3}$ and $2 \times 10^{-2} \mathrm{MeV}$, the solution spectrum was increased with increasing iterations, while between $4 \times 10^{-4}$ and $3 \times 10^{-3} \mathrm{MeV}$ the spectrum was decreased with increasing iterations. Neither of these changes was justified from the physics of the problem and thus could be considered "unphysical structure" in the solution spectra. The 20 iteration case provided the best values because there was little "unphysical structure" in the solution, the standard deviation of the measured to calculated activities was close to the average of the input activity uncertainties, and the maximum deviation was close to the maximum uncertainty in the input activities.

Figure 13 shows the SAND-II solution uncertainty after 20 and after 40 Monte Carlo recycles. The uncertainty in most of the group values decreased slightly on going from 20 to 40 recycles, but there were a few groups whose uncertainty increased slightly. Another indication of the adequacy of the choice of Monte Carlo recycles is the printout of the mean values and standard deviations of the cross sections and activities from the recycle information. Agreement of these values with the a priori values indicates a large enough choice for the number of recycles. From this information, 20 recycles were considered large enough, but there did not seem to be a great deal of sensitivity to the number of recycles used. Oster ${ }^{(9)}$ gives a more complete discussion of the effects of the number of iterations and the number of recycles on the SAND-II solution. 


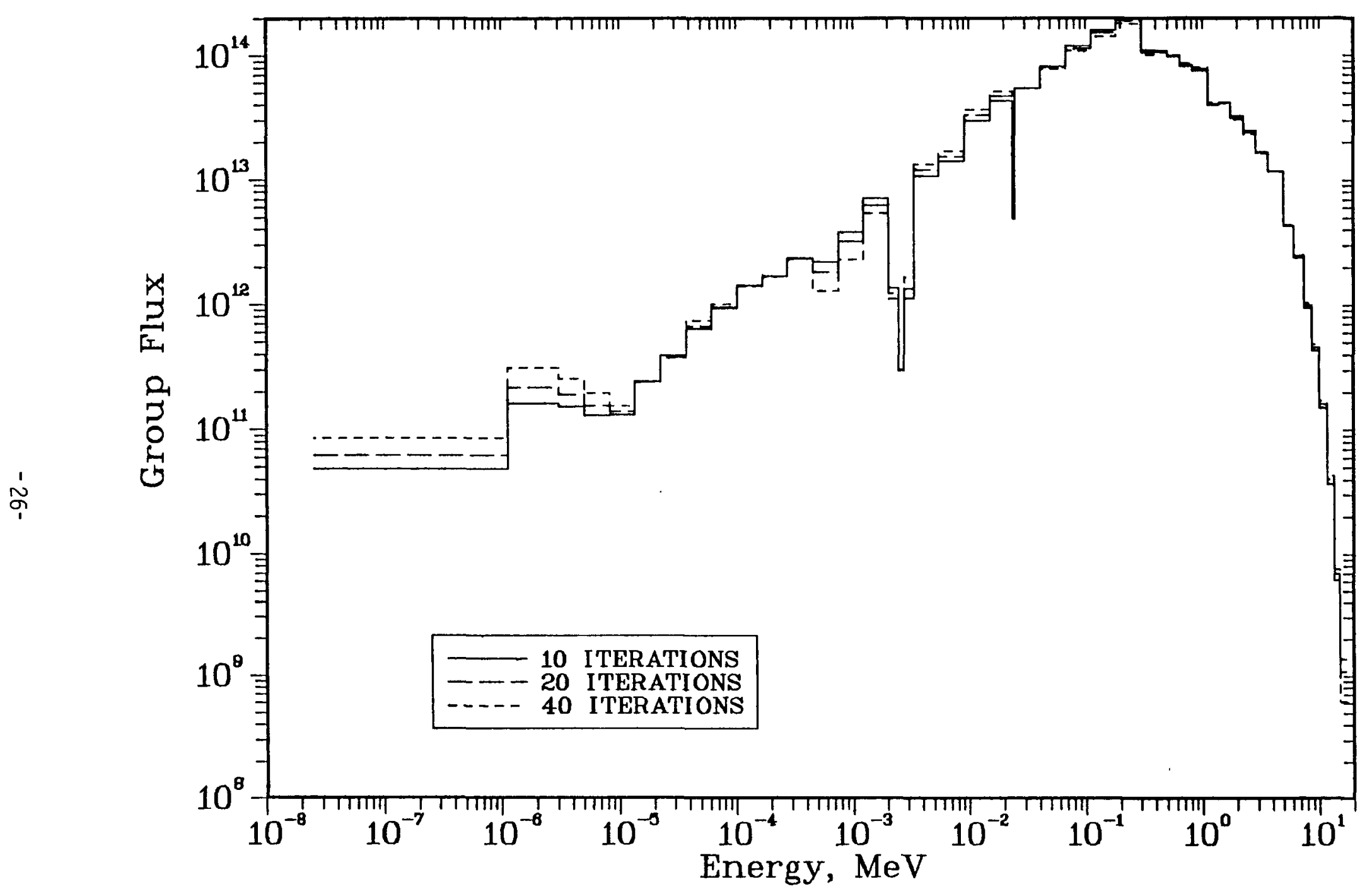

FIGURE 11. SAND-II solution spectrum variations with the number of iterations. 


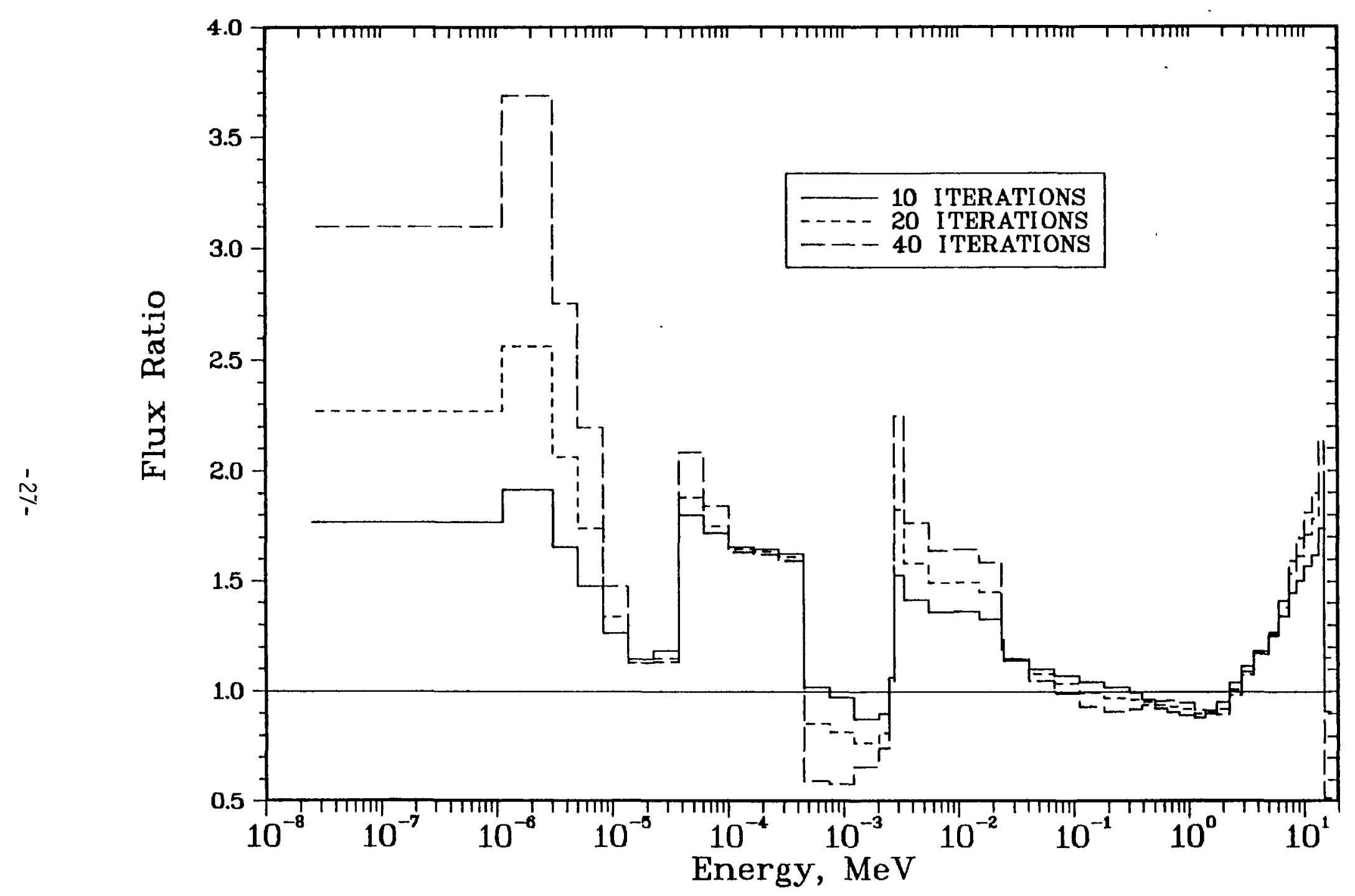

FIGURE 12. Ratio of solution spectrum to input spectrum with variations in the number of iterations. 


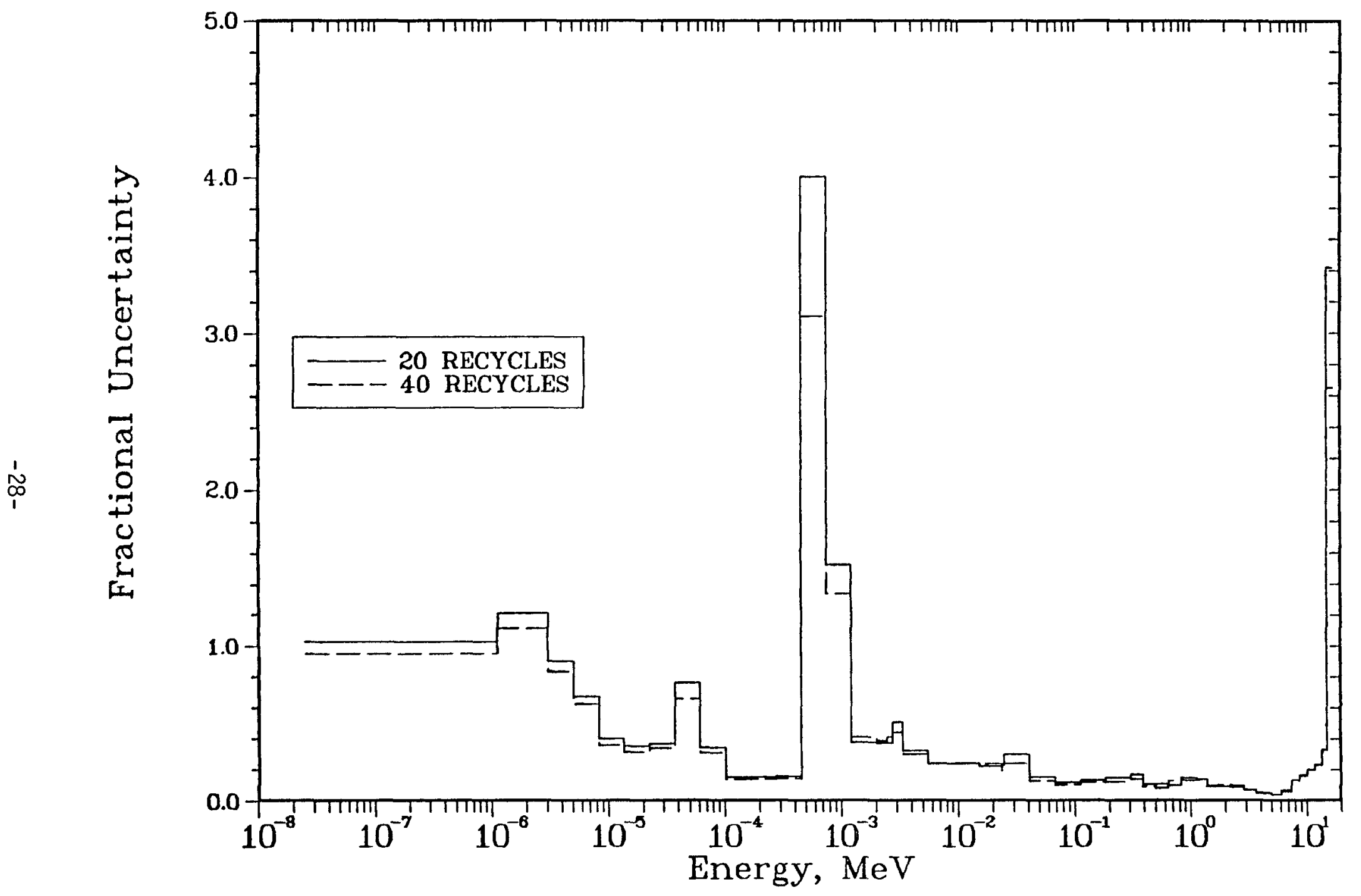

FIGURE 13. SAND-II solution uncertainty variations with the number of Monte Carlo recycles. 
The assumption that changing the SAND-II energy group structure from 620 to 47 groups did not affect the results was confirmed by repeating the base case with a 11 reactions in the 620 group structure. A 620-group cross section set based on the ENDF/B-IV dosimetry cross section file was used. For the most part the spectral adjustments for the 620-group analysis follow the 47-group SAND-II results. There was some deviation between the results below $10^{-3} \mathrm{MeV}$. No uncertainty analysis of the 620-group SAND-II results was performed.

To determine the sensitivity of SAND-II to the cross section set used, the same base case was repeated using the SAND-II 620-group evaluated reference cross section library. This library, routinely used for SAND-II runs at HEDL, contains cross sections that have been adjusted to attain integral consistency. A 47-group representation of the 620 -group solution spectrum was in much better agreement with the FERRET solution spectrum. Differences in the adjustments between this SAND-II run and FERRET are evident above $4 \mathrm{MeV}$, around $10^{-5} \mathrm{MeV}$, al though they are much less than in the original SAND-II run. Zijp (13) reported similar differences in the SAND-II adjustments of the benchmark neutron fields, CFRMF, $\Sigma \Sigma$, and STEK using ENDF/B-IV and a SAND-II evaluated reference cross section library. His spectra indicate much sharper adjustments in the energy region between $10^{-1}$ and $10^{-6} \mathrm{MeV}$ using the ENDF/B-IV cross section library and major differences in the spectral adjustments above $10 \mathrm{MeV}$. These studies indicate that the choice of cross section set can have significant effects on the SAND-II solution.

\section{FERRET}

Since the a priori flux uncertainty and short-range correlations reflect the degree of confidence placed in the a priori spectrum, it is important to identify their effects on the solution spectrum and uncertainty. Figures 14 and 15 show the solution spectra flux ratio and uncertainty for three different combinations of the strength and range of the a priori flux short-range correlations. The range of the correlation was varied from 3 to 5 groups and the strength of the correlation was varied from 0.9 to 0.5 . The effects of the correlations are clearly illustrated in the groups surrounding the ${ }^{59} \mathrm{Co}(n, y)$ resonance at about $10^{-4} \mathrm{MeV}$. Since this was a large response confined primarily to one group, the effect of the short-range correlations was to extend the flux adjustments in the group with the response to other nearby groups in a nearly symmetrical manner. When the range of the correlation was expanded from 3 to 5 groups, the adjustments made in the spectrum were greater and extended over 


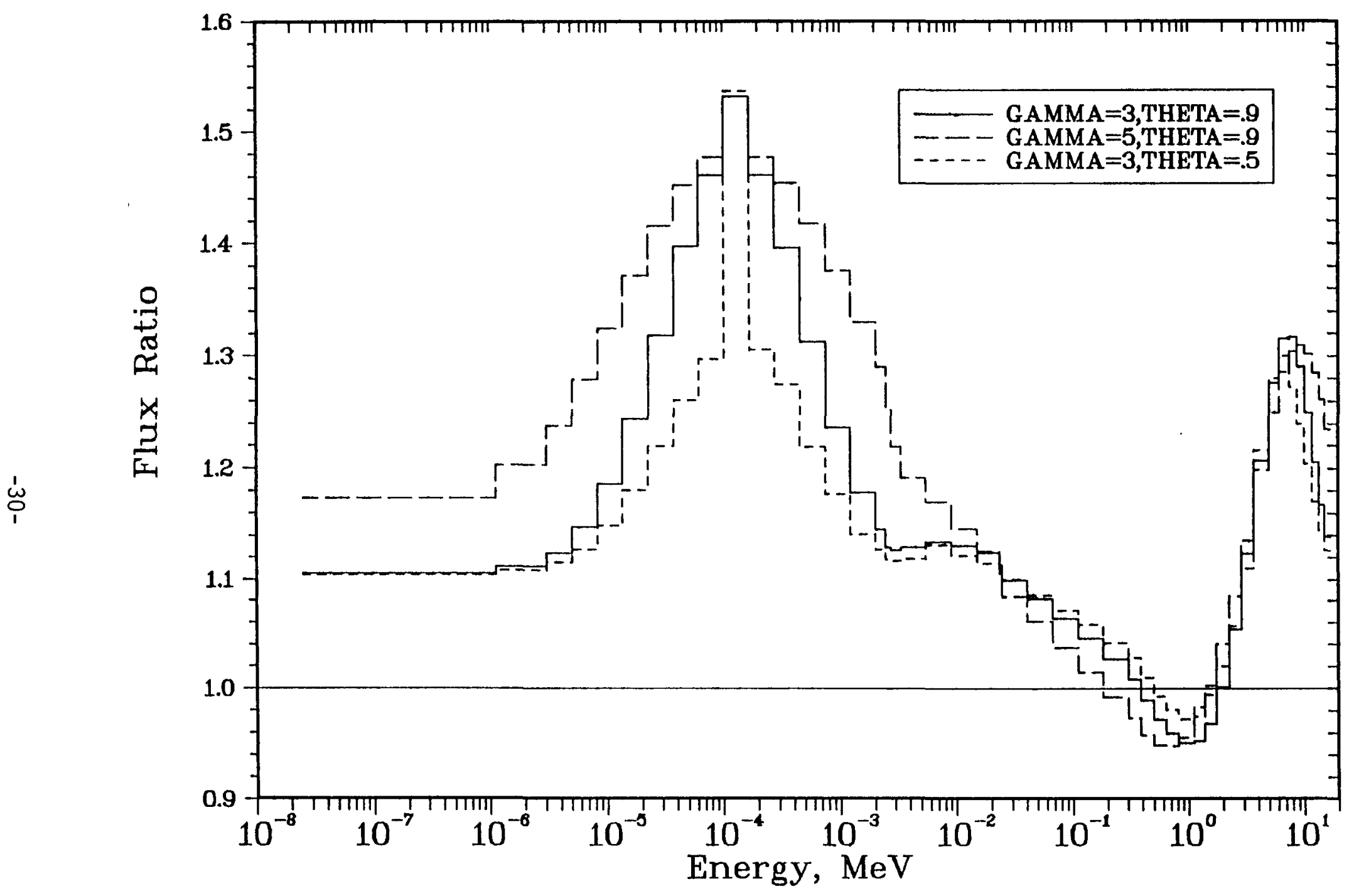

FIGURE 14. Ratio of solution spectrum to input spectrum for FERRET with variations in the input spectrum short range correlations. 


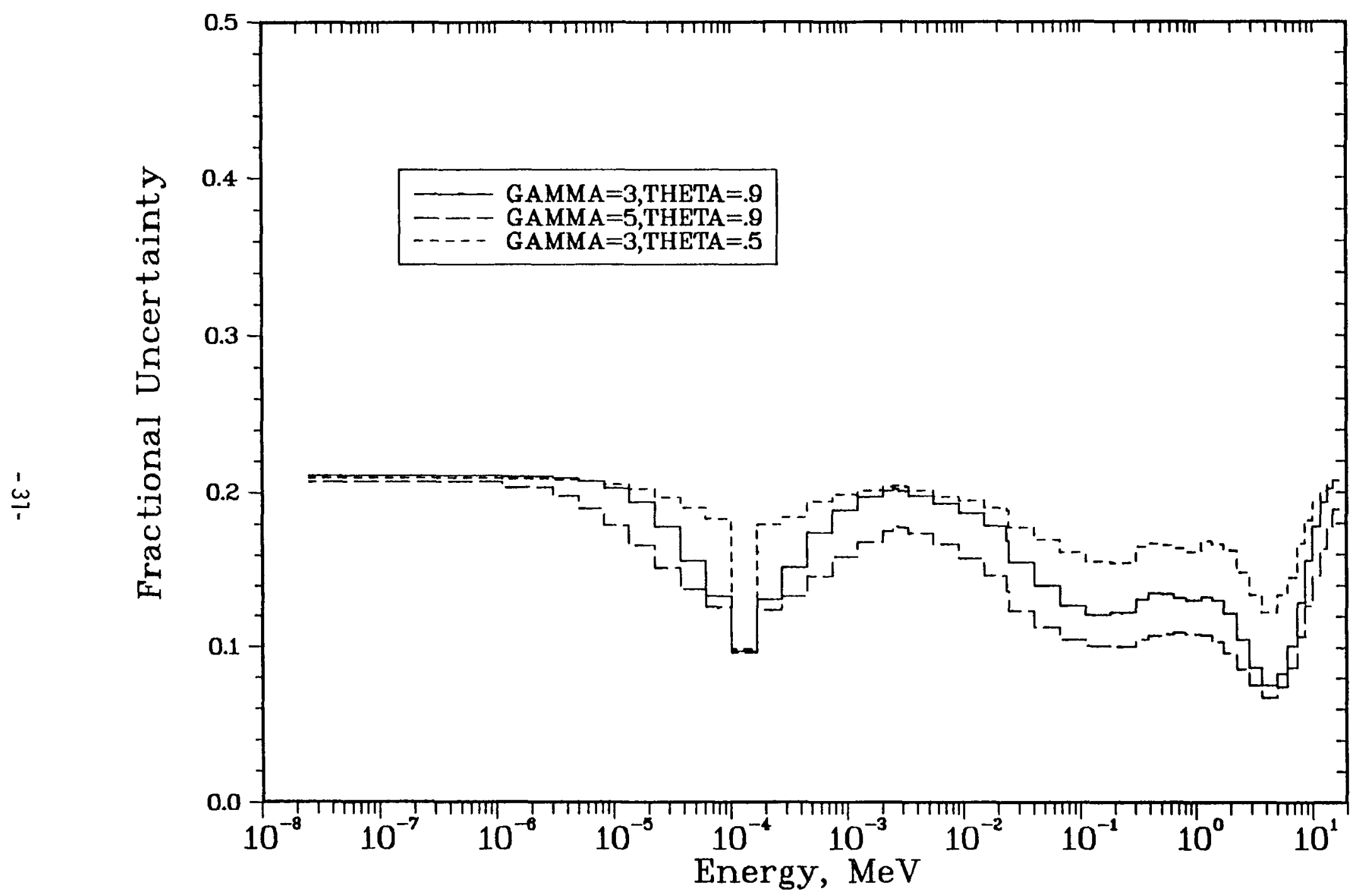

FIGURE 15. FERRET solution spectrum uncertainty with variations in input spectrum short range correlations. 
more groups. When the strength of the correlation was decreased from 0.9 to 0.5 , then the adjustments made in the spectrum were smaller and extended over fewer groups. These changes were less distinguishable in regions of overlapping detector response where the response was extended over several groups.

This same effect can be seen in the uncertainty plots in Figure 15 near the ${ }^{59} \mathrm{Co}(n, \gamma)$ resonance. The "rigidity" of the a priori spectrum has a greater effect on the uncertainty in regions of overlapping response. The stronger and longer-range correlations resulted in lower uncertainty estimates.

The actual values used for the a priori spectrum were a normalization uncertainty of $10 \%$ along with an additional group-by-group uncertainty of $20 \%$. Thus, the combined a priori spectral uncertainty was $22.3 \%$. Nearly a 11 (90\%) of the $20 \%$ uncertainty was assumed to be short range correlated over a range of 3 groups. The uncertainty values were chosen conservatively; i.e., large enough that they would not significantly affect the solution. It is possible for FERRET to give a wrong solution if the actual solution is outside the range of uncertainty on the input spectrum. The correlations were also chosen conservatively. The EBR-II spectrum consists basically of a fission spectrum and a slowing down region. Except for absorption resonances, such as $\mathrm{Na}$, the spectrum is a relatively smooth one. Therefore, as long as the physical structure is included in the input spectrum, neighboring groups should be somewhat correlated with each other.

Both SAND-II and FERRET require uncertainty estimates and correlations on the dosimeter cross sections. To determine the effects of these correlations on the solution and uncertainty, FERRET was run with the same correlations used in SAND-II. The GAVE structure used in SAND-II consists of 15 intervals, with complete correlation of the group cross section values with in each GAVE interval and no correlation between the groups in different GAVE intervals. This was implemented in FERRET by specifying 15 correlation components for each cross section corresponding to the 15 GAVE intervals. For each component a normalization uncertainty was used that was equal to the GAVE uncertainty estimate for that interval. All other uncertainties were set to zero so that all the groups in each interval would be completely correlated. 
The solution spectrum for this case was almost identical (within 1\%) to the solution with the original correlations. The uncertainty values, figure 16 , were also very close to the original values. The uncertainty at the ${ }^{59} \mathrm{Co}(n, \gamma)$ resonance is slightly larger while the uncertainty between 0.1 and $7 \mathrm{MeV}$ is slightly smaller. In the latter region the GAVE intervals contain only one or two groups. This has the effect of allowing a finer adjustment of the cross sections in this region, resulting in slightly lower uncertainties. Since the ${ }^{59} \mathrm{Co}(n, \gamma)$ response is located in the middle of a very wide GAVE interval, the cross section correlations are much stronger than the FERRET short range correlations, resulting in slightly larger uncertainty values. These results indicate that the correlations on the cross sections have little effect on the final solution and uncertainty in comparison with the correlations on the a priori flux.

\section{SIMPLE CASES TO CHECK UNCERTAINTY CALCULATIONS}

\section{$\underline{\text { SAND-I I }}$}

Since the SAND-II algorithm includes a smoothing weight function, few-group problems would not be valid. The simplest test that allowed proper code operation was to use a single simulated reaction with the cross section limited to one broad GAVE interval. Because the Monte Carlo variations are based on the GAVE structure, this simulated reaction gives uncertainty values that can be checked by hand. The results of several simple tests are shown in Table 3, where various choices of the uncertainty on the cross section and activity were used. The agreement between the SAND-II calculated spectral uncertainty and that predicted by simple hand calculations indicates that the SAND-II Monte Carlo routine gives valid results for uncomplicated problems in regions of strong non-overlapping response. 


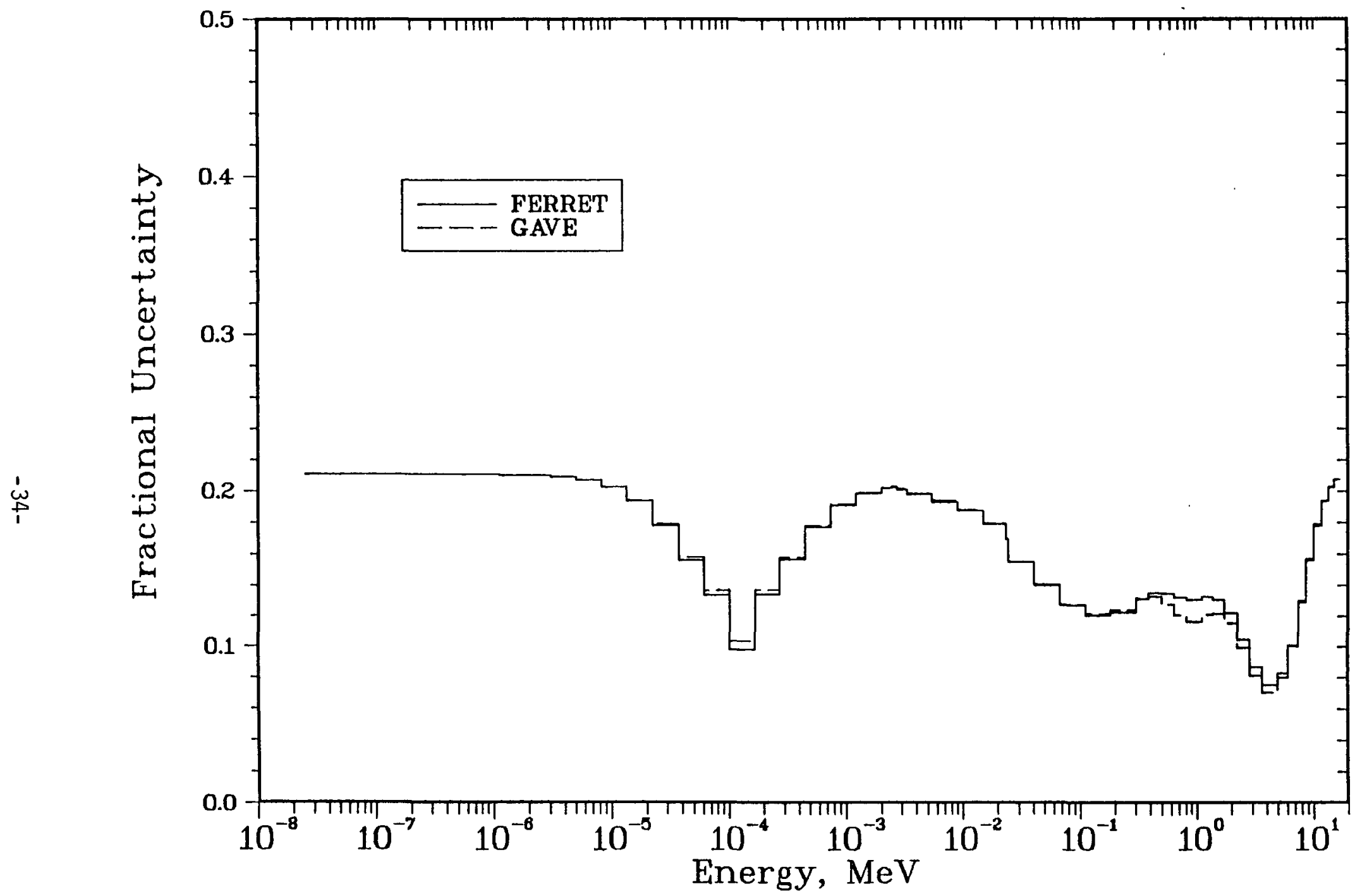

FIGURE 16. FERRET solution spectrum uncertainty with variations of the reaction cross section correlations. 
TABLE 3

RESULTS OF SIMPLIFIED SAND-I I UNCERTAINTY PROBLEMS

Input Uncertainty (Percent)

Cross Section Activity

0 .

10.

10.
10.

1.

10.
Calculated Spectrum Uncertainty

SAND-II Exact

$11.86 \quad 10.00$

$10.18 \quad 10.05$

$14.78 \quad 14.14$

\section{FERRET}

The FERRET algorithm for calculating the final covariance matrix is based on a formal derivation whose validity has been analytically demonstrated. ${ }^{(3)}$ In addition, the ability of this algorithm to exactly calculate the solution uncertainties (the diagonal elements of the final covariance matrix) has been verified with numerical examples. This algorithm has been shown to give exact values for the solution uncertainties in simple one and two group problems and in more complicated problems. Approximations enter the FERRET uncertainty calculation only for problems that are very weakly determined and thus have large a priori uncertainties. ${ }^{(3)}$ For these cases, however, a precise uncertainty estimate is usually not needed. Rather, one is usually only trying to distinguish a $100 \%$ uncertainty from a $10 \%$ uncertainty or a $1000 \%$ uncertainty.

\section{SUMMARY OF PERFORMANCE TEST RESULTS}

The results of the performance of SAND-II and FERRET under varying conditions revealed several operating characteristics and possible problems with each code.

1. The SAND-II iterative process may introduce unphysical structure in the solution spectrum, especially in the regions of detector resonances. (13) This structure consists of unlikely adjustments in neighboring groups that may cancel in the calculation of integral quantities such as total flux, but could have a large effect on integral parameters with significant response in the region of these adjustments. 
2. For certain conditions, the SAND-II Monte Carlo error analysis may give extremely large uncertainty values in regions of little or no detector response because of the nonconvergence of some of the recycle solutions.

3. SAND-II may give unjustifiably small uncertainty values in regions of little or no detector response.

4. The FERRET solution and uncertainty depend on the proper choice of the input spectrum uncertainties and correlations.

5. The FERRET and SAND-II solutions and uncertainties basically agree in the regions of strong response from a single detector, but deviations were found in regions of overlapping response or of little response.

6. The SAND-II solution and uncertainty were much more sensitive to the choice of dosimeter reactions, spectral coverage and cross section library.

\section{CONCLUSIONS}

This comparison of SAND-II and FERRET illustrated the major advantages and disadvantages of each code from both a technical and operational viewpoint. The FERRET code was judged to be an improvement over SAND-II based on the following conclusions:

1. FERRET has a rigorous theoretical foundation that provides a unique, most likely, solution and uncertainty, and makes explicit the assumptions upon which the solution is based.

2. The credibility of the FERRET solution can be inferred directly from the credibility of the input data. 
3. Conservative choices can be made in the input flux uncertainty and correlations for FERRET that allow conservative solution uncertainty estimates.

4. FERRET can provide a full covariance matrix for the solution spectrum that allows accurate uncertainty treatment for quantities derived from the spectrum.

5. The future availability of cross section covariance files in ENDF/B evaluations will allow more accurate uncertainty calculations using FERRET.

6. FERRET has the capability of simultaneous adjustment of spectra in different locations that should provide more consistent results. However, simultaneous solutions limit the number of groups to less than 620 due to computer storage 1 imitations. For spectrum adjustment in $\approx 50$ groups, care must be taken to weight the effective cross sections appropriately.

\section{REFERENCES}

1. G. L. Guthrie and R. L. Simons, "Analysis and Extension of the SAND-II Code", LWR Pressure Vessel Irradiation Surveillance Dosimetry, Quarterly Report, July-September 1977, HEDL-TME 78-4, Hanford Engineering Development Laboratory, Richland, WA (1978).

2. F. Schmittroth, "Varied Applications of a New Maximum-Likelihood Code With Complete Covariance Capability", in A Review of the Theory and Application of Sensitivity and Uncertainty Analys is, edited by C. R. Weisbin, et aT., ORNL/RSIC-42, Proceedings of a Seminar-Workshop, Oak Ridge National Laboratory, Oak Ridge, TN (August 22-24, 1978).

3. F. Schmittroth, A Method for Data Evaluation With Log-Normal Distributions, HEDL-SA-1742, Hanford Engineering Development Laboratory, Richland, WA (December 1978).

4. F. Schmittroth and J. A. Rawlins, Dosimeter Based Adjustments of FastNeutron Spectra, HEDL-SA-1480, Hanford Engineering Development Laboratory, Richland, WA (1978).

5. F. Schmittroth, FERRET Data Analys is Code, HEDL-TME 79-10, Hanford Engineering Deve Topment Laboratory, Richland, WA (September 1979). 
6. E. P. Lippincott and I. A. Ulseth, "High Flux Fluence Measurements in Fast Reactors," Proc. Second ASTM-EURATOM Symposium on Reactor Dosimetry: Dosimetry Methods for Fuel, Cladding and Structural Materials, October 37, 1977, NUREG/CP0004, Vol. I, pg. 271, Palo A1 to, CA.

7. W. N. McElroy and S. Berg, A Computer-Automated Iterative Method for Neutron Flux Spectra Determined by Foil Activation, AFWL-TR-67-41, VoI. II, Äir Force Weapons Laboratory, KirtTand Air Force Base, Albuquerque, NM (September 1967).

8. S. Berg, Modifications of SAND-II, BNWL-855, Battelle Memorial Institute/ Pacific Northwest Laboratory, Richland, WA (August 1968).

9. C. A. Oster, W. N. McElroy, R. L. Simons, E. P. Lippincott and G. R. Odette, A Modified Monte Carlo Program for SAND-I I with Solution Weighting and Error Analys is, HEDL-TME 76-60, Hanford Engineering Development Laboratory, Richland, WA (August 1976).

10. C. R. Weisbin, J. H. Marable, J. L. Lucius, E. M. Oblow, F. R. Mynatt, R. W. Peelle and F. G. Perey, Application of FORSS Sensitivity and Uncertainty Methodology to Fast Reactor Benchmark Analys is, ORNL/TM-5563, Oak Ridge National Laboratory, Oak Ridge, TN (Dec. 1976).

11. R. A. Ander 1, Y. D. Harker and F. Schmittroth, "Neodymium, Samarium and Europium Capture Cross-Section Adjustments Based on EBR-II Integral Measurements", Program of the International Conference on Nuclear Cross Sections for Technology, University of Tennessee, Knoxville, TN, October 22-26, 1979, Bulletin of the American Physical Society, Vol. 24, No. 7 (September 1979).

12. F. Schmittroth and D. W. Wootan, Covariances for Dosimeter Cross Sections, HEDL-TC-1588, Hanford Engineering Development Laboratory, Richland, WA (December 1979).

13. W. L. Zijp, J. H. Baard and H. J. Nolthenius, "Comparison of Neutron Spectrum Unfolding Codes," RCN-77-111, Reactor Centrum Nederland, Petten (1977).

Acknowledgment

The authors acknowledge the help of $R$. L. Simons in providing the 620 -group SAND-II analyses. 


\section{APPENDIX A}

WELL- AND ILL-DETERMINED COMPONENTS

OF AN UNFOLDED NEUTRON SPECTRUM

By a sultable diagonalization, one can decompose an unfolded neutron spectrum into two components, a well-determined component $\phi_{\text {we } 11}$ and an i11-determined component $\phi_{i 11}$. Although of little practical consequence, this decomposition does afford some insight into the nature of adjusted solutions.

The main point is that for a specific adjustment problem and a corresponding solution, it is possible to uniquely decompose the solution $\phi(E)$ into two functions

$$
\phi(E)=\phi_{\text {we11 }}(E)+\phi_{i 11}(E) \text {. }
$$

The first component $\phi_{\text {we } 11}$ is uniquely determined by the reaction rates used to obtain the unfolded solution. Thus, any solution $\phi(E)$ that gives the correct reaction rate values will have the same well-determined component as any other such solution. On the other hand, the ill-determined component is completely undetermined by the reaction rate measurements. In fact, for any reaction used in the adjustment analysis, the reaction rate calculated from the $i 11$-determined flux $\phi_{i 11}$ will of necessity be nearly zero.

Mathematically, this decomposition can be made by diagonalizing the matrix $A^{t} M_{f}^{-1} A$ where the $A$ matrix relates the vector $\vec{R}$ of reaction rate measurements to the flux vector $\vec{\phi}$. In a multigroup representation, we write

$$
R_{\alpha}=\sum_{g} A_{\alpha g} \phi_{g}
$$

where the matrix elements $\left\{A_{\alpha g}\right\}$ are obviously just the multigroup cross sections. The covariance matrix $M_{f}$ represents reaction rate covariances.

The details are beyond the scope of the present discussion. However, some results are illustrated in Figure $A 1$. The upper plot shows the welldetermined component for three adjusted spectra discussed in this report; a spectrum adjusted by SAND-II, a spectrum adjusted by FERRET using the same initial spectrum as SAND-II, and a spectrum adjusted by FERRET where 

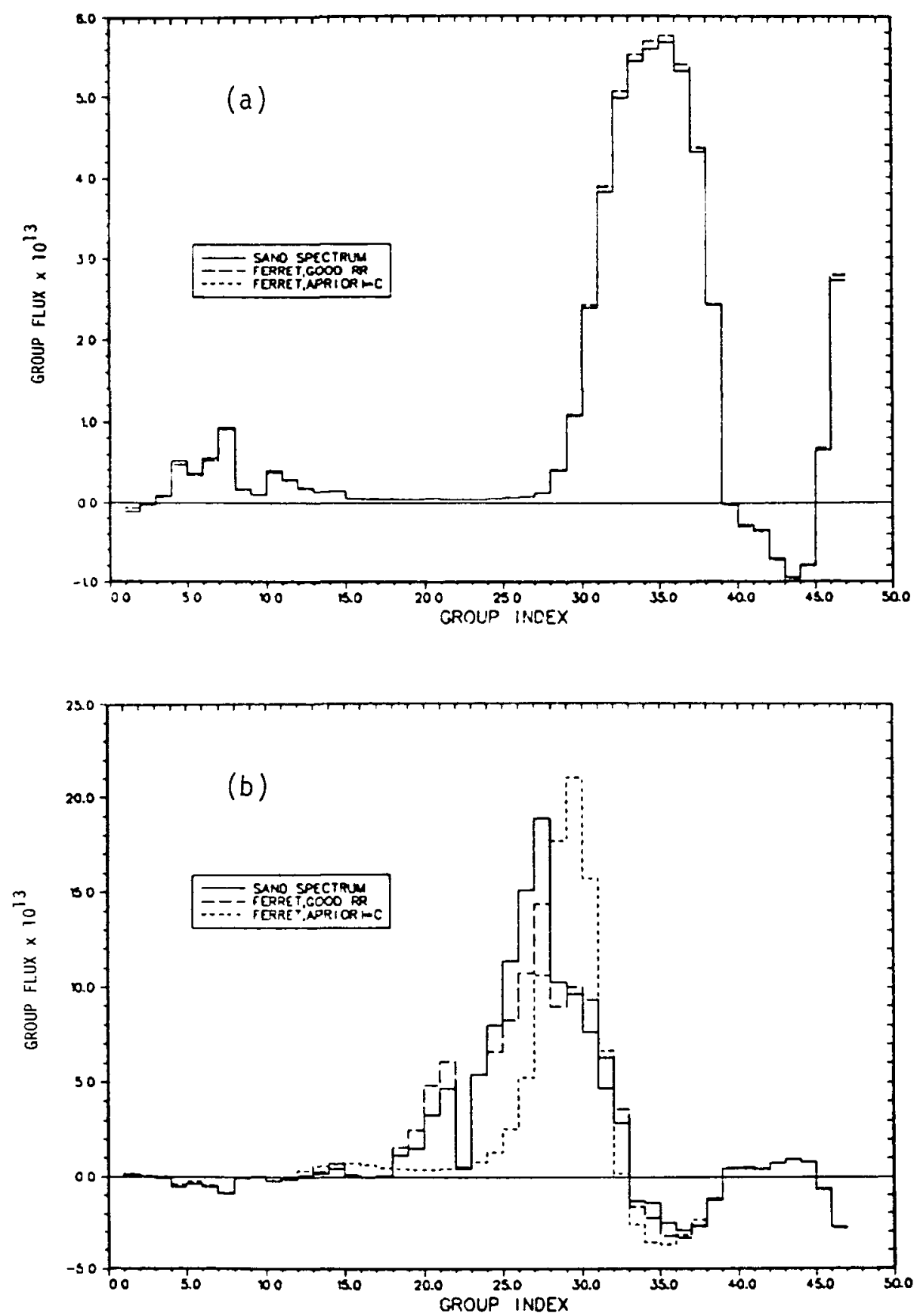

FIGURE A1. Decomposed components of adjusted neutron spectra.

(a) We 17-determined flux component

(b) 111-determined flux component 
the a priori spectrum was a constant group flux. (The group index on the plots is defined so that neutron energy increases from left to right.) As they should be, the well-determined components are nearly identical for all three solutions. Any differences in the adjusted solutions must appear in the ill-determined components.

The ill-determined components show significant differences that explicitly demonstrate the non-uniqueness in the adjusted solutions. The components for SAND-II and the first FERRET solution are remarkably similar and undoubtedly reflect the use of the same initial spectrum. Nevertheless, near a group index of 27 even these two components differ by $40 \%$. Since the well-determined component is small in this region, the complete solution $\phi(E)$ must also differ by $40 \%$. The solution obtained from FERRET with a constant a priori flux differs more radically even though it still gives the correct reaction rates (it has the same well-determined component).

The significance of these decompositions is in the explicit demonstration of a component $\phi_{i 11}$ that is completely independent of the reaction rate data. Any calculation that depends on $\phi_{i 11}$ necessarily relies on subsidiary information that is in addition to the reaction rate measurements. Furthermore, the accuracy of such calculations must also rely on the uncertainties or reliability of such subsidiary data (trial spectra, smoothing constraints, etc.). In the present example, for instance, less than half of the total flux is contained in the well-determined component. One could attain an alternate solution with less than one-half of the total flux, which also reproduced the reaction rates, by setting $\phi_{i 11}$ to zero. This statement is somewhat misleading because such a solution would have negative flux values in some groups. Nevertheless, the total flux for two of these solutions, the SAND-II case and the FERRET case with a constant a priori flux, differs by nearly $20 \%$.

Finally, we note that any algorithm that determines uncertainties on the unfolded solution by propagating only reaction rate and cross section uncertainties is subject to error. The component $\phi_{i 11}$ is an important part of the final solution and its uncertainties must depend strongly on both the subsidiary conditions needed to obtain $\phi_{i 11}$ as well as any uncertainties in these subsidiary conditions. 


\section{APPENDIX B}

DOSIMETER RESPONSE FUNCTIONS

IN AN EBR-II SPECTRUM 


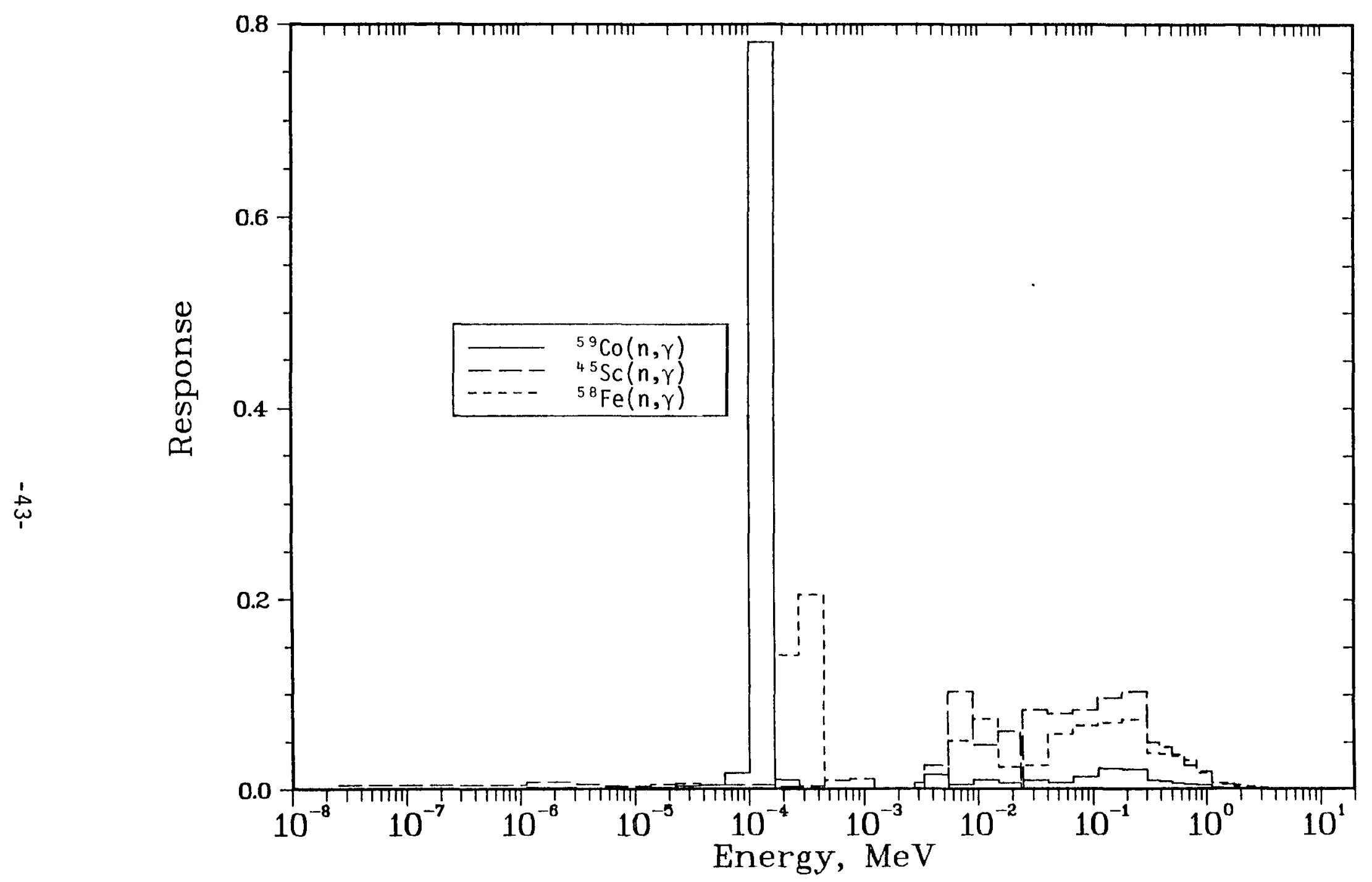

FIGURE B1. Activation reactions response in EBR-II midplane location.

Product of cross section and flux spectrum, normalized to unit area. 


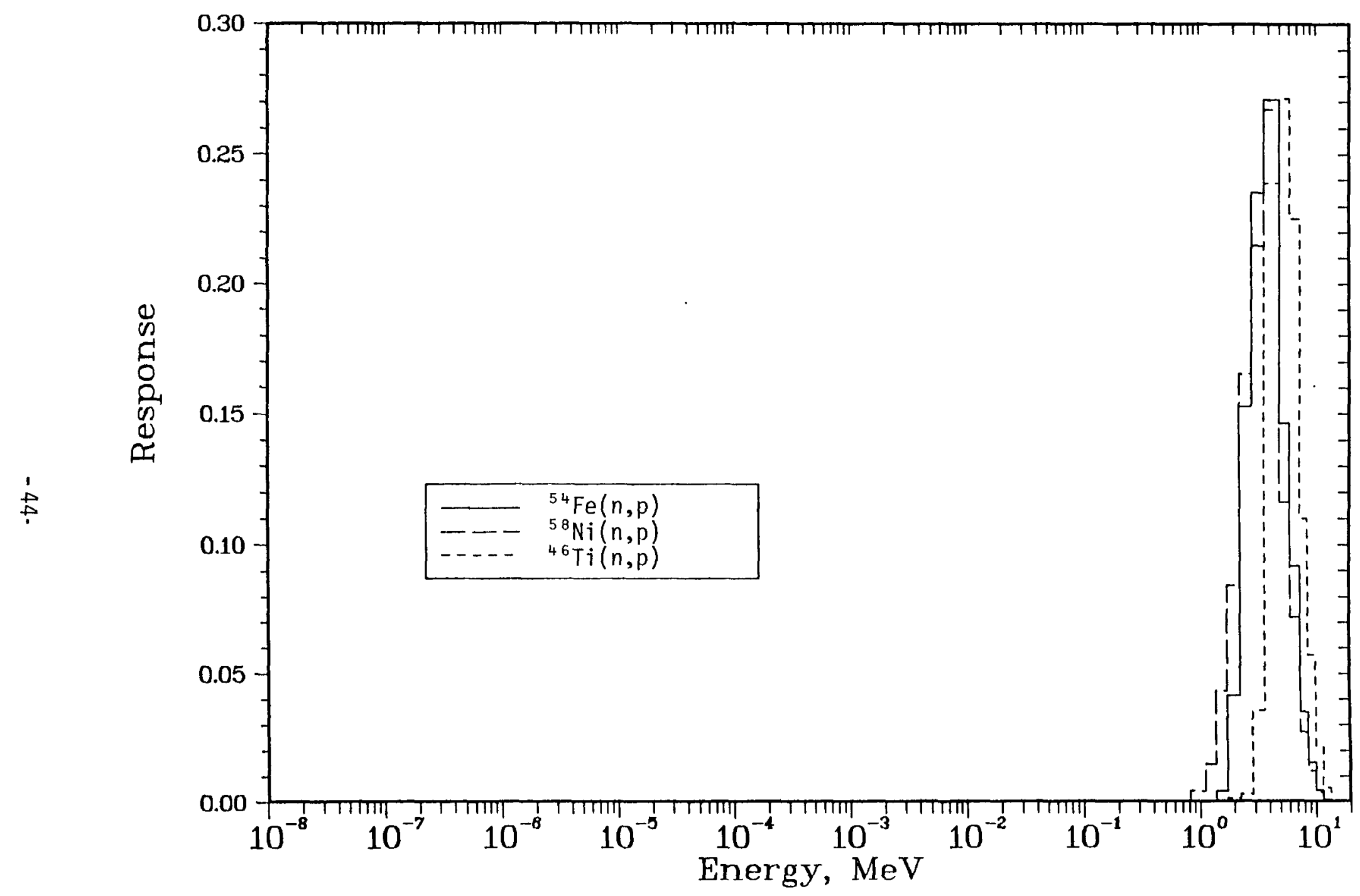

FIGURE B2. Threshold reactions response in EBR-II midplane location.

Product of cross section and flux spectrum, normalized to unit area. 


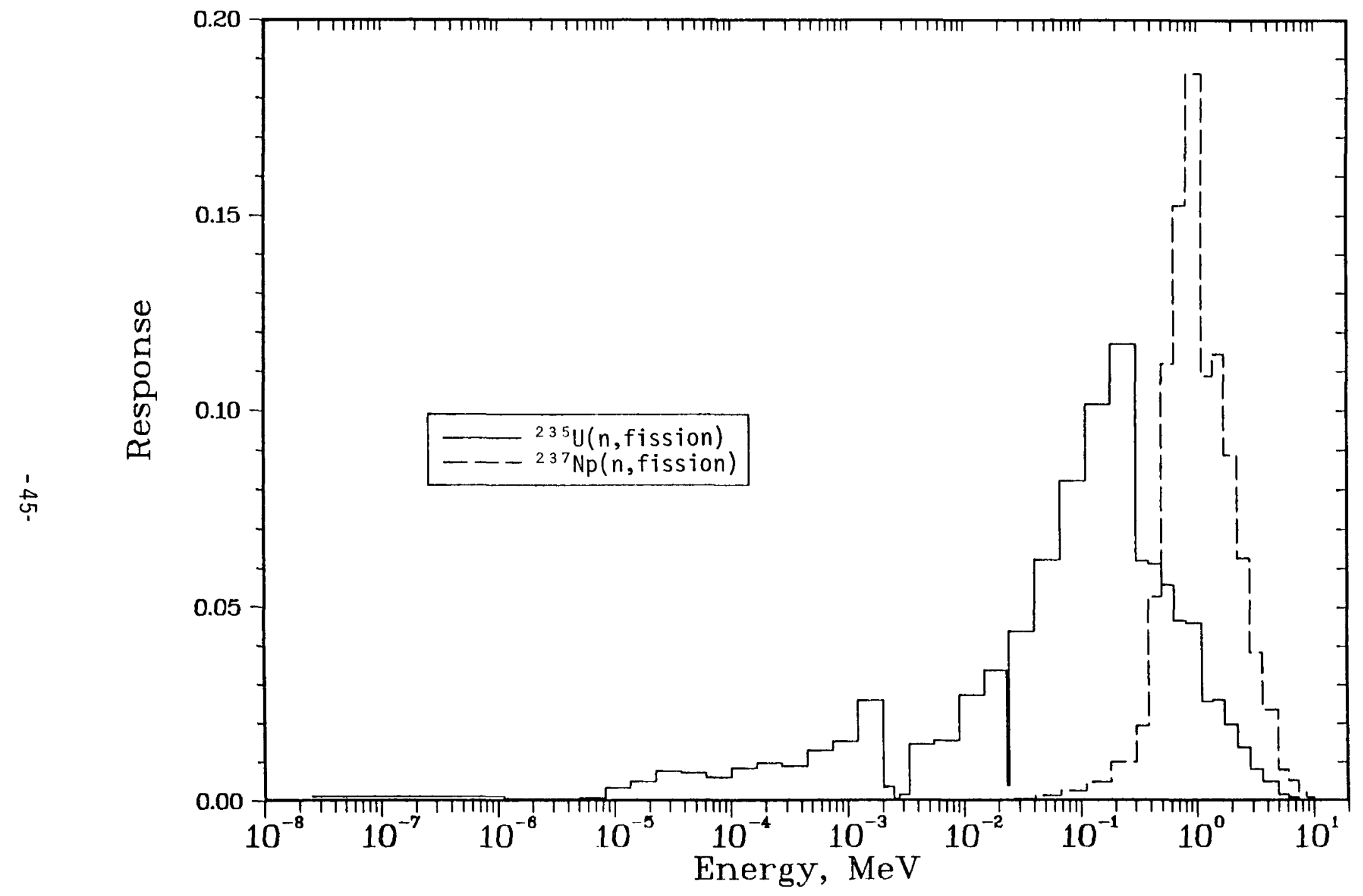

FIGURE B3. Fission reactions response in EBR-II midplane location.

Product of cross section and flux spectrum, normalized to unit area. 


\section{DISTRIBUTION}

DOE/FFTFPO (7)

Director (5)

Reactor Safety Field Office

T. L. King

DOE/NPD-HQ (2)

Director

Chief, Quality Assurance and Standards and Operational Safety Branch

HEDL (38)

$\begin{array}{ll}\text { RA Bennett } & \text { W/B-43 } \\ \text { WL Bunch } & \text { W/B-47 } \\ \text { LL Carter } & \text { W/B }-47 \\ \text { JW Daughtry } & \text { W/B-45 } \\ \text { KD Dobbin } & \text { W/B-45 } \\ \text { DG Doran } & \text { W/A-57 } \\ \text { GL Guthrie } & \text { W/C-39 } \\ \text { RA Harris } & \text { W/B-45 } \\ \text { RL Knecht } & \text { W/A-40 } \\ \text { EP Lippincott } & \text { W/C-39 } \\ \text { AT Luksic } & \text { W/B-47 } \\ \text { WN MCElroy } & \text { W/C-39 } \\ \text { WJ MCShane } & \text { W/B-12 }\end{array}$

$\begin{array}{ll}\text { FS Moore } & \text { W/B-47 } \\ \text { RJ Morford } & \text { W/B-47 } \\ \text { EW Pottmeyer, Jr. } & \text { W/B-63 } \\ \text { JA Rawlins } & \text { W/B-45 } \\ \text { RB Rothrock } & \text { W/B-45 } \\ \text { RE Schenter } & \text { W/A-4 } \\ \text { F Schmittroth (2) } & \text { W/A-4 } \\ \text { RL Simons } & \text { W/A-57 } \\ \text { WP Stinson } & \text { W/B-47 } \\ \text { AD Wilcox (2) } & \text { W/B-47 } \\ \text { DW Wootan (2) (10) } & \text { W/B-45 } \\ \text { Central Files (170 } & \text { W/C-715 } \\ \text { Tech. Services (2) } & \text { W/C-715 }\end{array}$

Distr. 1 


\section{DISTRIBUTION (cont.)}

Argonne National Laboratory

9700 South Cass Avenue

Argonne, Illinois 60439

L. R. Greenwood

D. L. Smith

Brookhaven National Laboratory Upton, New York 11973

B. Magurno

EG\&G Idaho, Inc.

P. 0. Box 1625

Idaho Falls, Idaho 83401

R. A. Ander 1

Y. D. Harker

Electric Power Research Institute 3412 Hillview Avenue

P. 0. Box 10412

Palo Alto, California 94304

Odelli Ozer

Los Alamos Scientific Laboratory

P. 0. Box 1663

Los Alamos, New Mexico 87545

L. Stewart (MS-243)

U. S. Department of Commerce National Bureau of Standards Center for Radiation Research Washington, DC 20234

E. D. McGarry (532.04)

Oak Ridge National Laboratory P. 0. Box X

Oak Ridge, Tennessee 37830

R. W. Peelle

F. G. Perey

C. R. Weisb in

Purdue University

School of Nuclear Engineering

West Lafayette, Indiana 47907

R. H. Johnson

Distr. 2 


\section{DISTRIBUTION (cont.)}

University of Missouri

Department of Nuclear Engineering

Columbia, Missouri 65201

(1)

Dr. Walter Meyer

Westinghouse Electric Corporation

Fusion Power Systems Department

P. 0. Box 10468

Pittsburgh, Pennsylvania 15236

L. Green 